\title{
Using spectroscopic data to disentangle stellar population properties ${ }^{\star}$
}

\author{
N. Cardiel ${ }^{1,2}$, J. Gorgas ${ }^{2}$, P. Sánchez-Blázquez ${ }^{2}$, \\ A. J. Cenarro ${ }^{2}$, S. Pedraz ${ }^{1,2}$, G. Bruzual ${ }^{3}$, and J. Klement ${ }^{4}$
}

1 Calar Alto Observatory, CAHA, Apdo. 511, 04004, Almería, Spain

2 Departamento de Astrofísica, Facultad de Físicas, Universidad Complutense de Madrid, 28040 Madrid, Spain

3 Centro de Investigaciones de Astronomía (CIDA), Apartado Postal 264, Mérida 5101-A, Venezuela

${ }^{4}$ Institut für Astronomie, ETH Zentrum, SEC E3, Scheuchzerstrasse 7, 8092 Zürich, Switzerland

Received 11 February 2003 / Accepted 18 June 2003

\begin{abstract}
It is well known that, when analyzed in the light of current synthesis model predictions, variations in the physical properties of single stellar populations (e.g. age, metallicity, initial mass function, element abundance ratios) may have a similar effect in their integrated spectral energy distributions. The confusion is even worsened when more realistic scenarios, i.e. composite star formation histories, are considered. This is, in fact, one of the major problems when facing the study of stellar populations in star clusters and galaxies. Typically, the observational efforts have aimed to find the most appropriate spectroscopic indicators in order to avoid, as far as possible, degeneracies in the parameter space. However, from a practical point of view, the most suited observables are not, necessarily, those that provide more orthogonality in that parameter space, but those that give the best balance between parameter degeneracy and sensitivity to signal-to-noise ratio per $\AA$, $S / N(\AA)$. In order to achieve the minimum combined total error in the derived physical parameters, this work discusses how the functional dependence of typical line-strength indices and colors on $S / N(\AA)$ allows to define a suitability parameter which helps to obtain more realistic combinations of spectroscopic data. As an example, we discuss in more detail the problem of breaking the well known age-metallicity degeneracy in relatively old stellar populations, comparing the suitability of different spectroscopic diagrams for a simple stellar population of solar metallicity and of $12 \mathrm{Gyr}$ in age.
\end{abstract}

Key words. methods: data analysis - techniques: spectroscopic - galaxies: stellar content

\section{Introduction}

\subsection{Evolutionary synthesis modeling}

In order to predict the expected spectral energy distribution (SED) of simple stellar populations (chemically homogeneous and coeval stellar systems), it is possible to use first principles (e.g. initial mass function, star formation rate, stellar isochrones, element abundance ratios) to generate synthetic star systems. This technique, known as evolutionary synthesis modeling, has been widely employed to understand the origin and evolution of star clusters and galaxies (Crampin \& Hoyle 1961; Tinsley 1972, 1978, 1980; Tinsley \& Gunn 1976; Gunn et al. 1981; Bruzual 1983, 2002; Aragón-Salamanca et al. 1987; Charlot \& Bruzual 1991; Bruzual \& Charlot 1993; Arimoto \& Yoshii 1986, 1987; Guiderdoni \& Rocca-Volmerange 1987; Buzzoni 1989, 1995; Mas-Hesse \& Kunth 1991;

Send offprint requests to: N. Cardiel,

e-mail: ncl@astrax.fis.ucm.es

* Appendices A-C are only available in electronic form at http://www.edpsciences.org
Fritze-v Alvensleben \& Gerhard 1994; Cerviño \& Mas-Hesse 1994; Worthey 1994; Worthey \& Ottaviani 1997; Bressan et al. 1994; Chiosi et al. 1996; Tantalo et al. 1998; Milone et al. 1995; Leitherer \& Heckman 1995; Leitherer et al. 1996, 1999; Fioc \& Rocca-Volmerange 1997; Vazdekis et al. 1996, 1997, 2003; Vazdekis 1999; Mayya 1995, 1997; García-Vargas et al. 1998; Mollá \& García-Vargas 2000; Maraston 1998; Schiavon et al. 2000; Origlia \& Oliva 2000; Zackrisson et al. 2001; Thomas et al. 2003).

The reliability of model predictions has greatly increased as their developers include more realistic physical ingredients. However, as discussed by Charlot et al. (1996), there are still problems due to uncertainties in the theory of stellar evolution (e.g. post-main-sequence stages), the physics of stellar interiors (e.g. atomic diffusion, helium content, the temperature of the red giant branch), and the lack of complete stellar spectra libraries. It is important to note that although initially it is straightforward to predict spectroscopic indices from this type of models, the inherent problems associated to the SED libraries, either empirical or theoretical, have a non negligible influence in the line-strength predictions. For instance, 
empirical SED libraries constitute a coarse grained, and usually incomplete (specially for nonsolar metallicities and nonsolar abundance ratios) sampling of the atmospheric stellar parameter space, whereas theoretical libraries usually exhibit systematic discrepancies among themselves and when compared with observational data (e.g. Lejeune et al. 1997, 1998).

The use of empirical fitting functions (e.g. Gorgas et al. 1993, 1999; Worthey et al. 1994; Cenarro et al. 2002) can help to reduce substantially these effects (Worthey 1994; Vazdekis et al. 2003). They do not only allow the computation of line-strength indices for any given combination of input parameters, but the error in their predictions can be minimized with the use of a large set of stars. However, and since the empirical fitting functions only predict the value of a given line-strength feature for a fixed set of stellar atmospheric parameters, the shape of the spectrum that leads to such value is therefore unknown. To insert the fitting function predictions into the evolutionary synthesis models it is necessary to use the local continuum of each single star in the SED library as a reference continuum level. In this way it is possible to weight the luminosity contribution of each type of star, in the neighborhood wavelength region of each index, to obtain the final line-strength prediction.

In addition, there are also additional sources of biases in model predictions. Cerviño et al. (2000, 2001, 2002), and Cerviño \& Valls-Gabaud (2003) have thoroughly analyzed the impact of the actual discreteness of real stellar populations (see also Bruzual 2001, and references therein), the Poissonian dispersion due to finite populations in non-time-integrated observables, and the influence of the interpolations in time-integrated quantities, among others.

But far from being a discouraging situation, the recognition of all these problems is providing a solid understanding of the challenging task of modeling stellar populations. In this sense, the collective effort of many modelers (e.g. Leitherer et al. 1996) is giving strength to the idea that reliable and unbiased model predictions are starting to emerge.

\subsection{Physical parameter degeneracy}

Although spectroscopic data provide a direct way to analyze the integrated light of composite stellar systems, the predictions from simple stellar population synthesis models reveal that variations in the relevant physical properties of such systems may produce quite similar spectral energy distributions (SEDs). This conspiracy leads to undesirable degeneracies when passing from the observable space (e.g. that defined by line-strength indices and colors), to the parameter space (age, metallicity, initial mass function, etc.).

Among the best known examples of degeneracy we must highlight the one exhibited by age and metallicity in the study of relatively old stellar populations (O'Connell 1976, 1980, 1994; Aaronson et al. 1978; Worthey 1994; Faber et al. 1994). This outstanding problem drove many authors to seek spectral line-strength indices which were more sensitive to age than to metallicity and vice versa (e.g. Rose 1985, 1994; Worthey 1994). In this sense, Worthey (1994) introduced an interesting quantitative measure of the metal sensitivity of each index, computed as the partial derivatives $d \log ($ age $) / d \log (Z)$ around his model predictions for a 12 Gyr old stellar population with solar metallicity. Since then, large efforts have been focused toward the search for spectral features with very high (e.g. Fe4668) and very low (e.g. $\mathrm{H} \beta, \mathrm{H} \gamma$ ) metal sensitivities. However, this work has led to the use of individual and narrow absorption features (e.g. Jones \& Worthey 1995; Worthey \& Ottaviani 1997; Vazdekis \& Arimoto 1999) for which accurate measurements demand high signal-to-noise ratios. In addition, these spectral signatures are usually very sensitive to spectral resolution and, therefore, velocity dispersion.

\subsection{Compromise between orthogonality and errors}

It is important to note that since the problem is to break a degeneracy, in practice the real concern is how uncertain the requested physical parameters are when derived from a particular observable space. In this sense, two circumstances have to be carefully handled. The first is the orthogonality of the isoparameter lines in the observable space. As we have just mentioned, this is precisely the major concern of previous works. The second condition to be aware of is the propagation of the errors in the spectroscopic indices into the corresponding errors in the parameters. However, and as it is expected, narrow indices (better suited to provide more orthogonality) exhibit larger errors than broad spectral features, for a given signalto-noise ratio. Summarizing, orthogonality and small errors are magnitudes that can not be, a priori, simultaneously maximized. As a result, it seems clear that the most suited observable space will be that in which the two mentioned requirements are best balanced.

The relevance of finding this equilibrium can hardly be overemphasized, specially when one considers the important observational effort that is being (or is going to be) spent in ambitious spectroscopic surveys, like e.g. DEEP (Mould 1993; Koo 1995), EFAR (Wegner et al. 1996), CFRS (Lilly et al. 1995; Hammer et al. 1997), Sloan (York et al. 2000; Kauffmann et al. 2003), VLT-VIRMOS (Le Fèvre et al. 2000), SDSS (Bernardi et al. 2003). In all these type of surveys, a large amount of spectroscopic data is collected, although signalto-noise ratios and spectral resolution are typically moderate. These factors strengthen the need of a quantitative estimation of the reliability of the physical parameters derived from such spectroscopic studies.

With a clear practical sense, in this paper we explore the way to determine those combinations of spectroscopic observables that provide robust tools to constrain physical properties in stellar populations. For this purpose, we are going to assume that evolutionary synthesis model predictions are error free. Although, as we have discussed in Sect. 1.1, this is not at present the case, we want to concentrate in the problem of balancing errors and degeneracy. For this reason, model uncertainties and biases are out of the scope of this paper. In Sect. 2 we review and enlarge our previous work concerning random errors in line-strength indices, showing that a common functional dependence of final index errors on signal-to-noise 
ratio can be found for different index and color definitions. In Sect. 3 we obtain simple formulae to quantify total errors in the derived physical parameters when derived from spectroscopic measurements. As an illustrative example, we examine in more detail the age-metallicity degeneracy in Sect. 4, through the comparison of the suitability of different spectroscopic diagrams for a $12 \mathrm{Gyr}$ old simple stellar population with solar metallicity. We summarize the conclusions of this work in Sect. 5. Finally, some more technical aspects have been deferred to Appendices A-C.

\section{Errors and spectroscopic measurements}

\subsection{Error handling}

Random uncertainties and biases are inherently associated with the physical process of data acquisition. Random errors can be easily derived with the help of statistical methods. Unfortunately, the situation is not so simple when handling systematic effects, where a case by case solution must be sought. In practice, the aim is to obtain reliable quantitative constraints of the total random errors present in the data while having uncorrected systematic effects (if any) well within the range spanned by the former. For this to be the case, possible sources of systematic effects should be identified and alleviated during the measure process. In this paper we are assuming that this is actually the case, and for that reason we are exclusively focusing on the impact of random errors.

Although, as we have just mentioned, appropriate observational strategies can greatly help in reducing the sources of data biases, the unavoidable limited exposure time that can be spent in each target determines the maximum signal-to-noise ratio in practice achievable. The data reduction process, aimed to minimize the impact of data acquisition imperfections on the measurement of data properties with a scientific meaning for the astronomer, is typically performed by means of arithmetical manipulations of data and calibration frames. As a result, the initial random errors present in the raw scientific and calibration data are combined (and thus enlarged) and propagated throughout the reduction procedure.

In a recent paper, Cardiel et al. (2002) have discussed the benefits and drawbacks of different methods to quantify random errors in the context of data reduction pipelines. One of the conclusions of this work is that a parallel reduction of data and error frames is likely the most elegant and general approach, and, in some circumstances, the only way to proceed when observing or computing time demands are specially restrictive. It must be noted, however, that in order to apply this method to compute final errors, it is essential to prevent the introduction of correlation between neighboring pixels, which dangerously leads to underestimated errors. This problem arises when one performs image manipulations involving rebinning or noninteger pixel shifts of data, which is the case of those data reduction steps devoted to correct for geometric distortions, to produce a wavelength calibration into a linear scale, or to correct for differential refraction variations with wavelength, to mention a few. Fortunately, a modification in the way typical reduction processes operates can help to solve this problem.
Although we are not going to enter into details (we refer the interested reader to that paper), the key point is to transfer the responsibility of the most complex reduction steps to the analysis tools, which must manipulate data and error frames using a distorted system of coordinates, overriding the orthogonal coordinate system defined by the physical pixels in a detector.

\subsection{Random errors in spectroscopic measurements}

Once it can be assumed that reliable final random error estimates are available, and that, in comparison, systematic biases are not relevant, it is straightforward to obtain a quantitative estimate of the error in a given spectroscopic measurement. Since the information collected by detectors is physically sampled in pixels, the starting point in the analysis of a single spectrum will be the spectrum itself $S\left(\lambda_{i}\right)$ (with $i=1, \ldots, N_{\text {pixels }}$ ) and its associated random error spectrum $\sigma_{S}\left(\lambda_{i}\right)$. In the following discussion, we are assuming

$$
\begin{aligned}
\operatorname{cov}\left[S\left(\lambda_{i}\right), S\left(\lambda_{j}\right)\right]= & \left\langle S\left(\lambda_{i}\right) S\left(\lambda_{j}\right)\right\rangle-\left\langle S\left(\lambda_{i}\right)\right\rangle\left\langle S\left(\lambda_{j}\right)\right\rangle=0, \\
& \forall i, j \in\left[1, N_{\text {pixels }}\right], \quad i \neq j,
\end{aligned}
$$

i.e. errors are not correlated. From here, two different approaches can be followed (Cardiel et al. 1998; Cardiel 1999).

One possibility is to estimate numerically the effect of errors via Monte Carlo simulations. In practice, new instances of the spectrum, $\tilde{S}\left(\lambda_{i}\right)$, can be generated introducing Gaussian noise in each pixel using, for example,

$$
\tilde{S}\left(\lambda_{i}\right)=S\left(\lambda_{i}\right)+\sqrt{2} \sigma_{S}\left(\lambda_{i}\right) \sqrt{-\ln \left(1-r_{1}\right)} \cos \left(2 \pi r_{2}\right),
$$

where $r_{1}$ and $r_{2}$ are two random numbers in the range $r_{1}, r_{2} \in$ $[0,1)$. The unbiased standard deviation of all the measurements, performed over a sufficiently large number of simulated spectra, provides the final error.

Another method to estimate errors consists in the use of analytical formulae. In fact, Cardiel et al. (1998) and Cenarro et al. (2001) have already presented analytical expressions to compute errors in the $4000 \AA$ break $\left(\mathrm{D}_{4000}\right.$; defined by Bruzual 1983), and in atomic, molecular and generic indices. Interestingly, in the case of molecular indices, and when atomic and generic indices are measured in magnitudes, index errors can be derived by (see e.g. Appendix A in Cenarro et al. 2001)

$\sigma(M) \simeq \frac{c(M)}{S / N(\AA)}$,

where $\sigma(M)$ is the random error in the index $M, S / N(\AA)$ is the averaged signal-to-noise ratio per $\AA$ measured in the pixels included in all the bandpasses which define the considered index, and $c(M)$ is an index dependent constant. A similar expression can be deduced for the $\mathrm{D}_{4000}$ (Cardiel 1999), namely

$$
\sigma\left(\mathrm{D}_{4000}\right) \simeq \frac{\mathrm{D}_{4000}}{10} \frac{1}{S / N(\AA)} .
$$

However, in order to take advantage of the fact that the relative error in $\mathrm{D}_{4000}$ is exclusively a function of $S / N(\AA)$, it is more 
Table 1. Coefficients to estimate the expected random error in typical line-strength indices and colors, as a function of the mean signal-tonoise ratio per $\AA$, following Eq. (3). Note that these coefficients are valid for atomic indices when measured in magnitudes (i.e. as if they were molecular indices). For line-strength indices measured in $\AA$ see coefficients in Table 1 in Cardiel et al. (1998).

\begin{tabular}{lc|lc|lc}
\hline \hline Index & $c$ & Index & $c$ & Color & $c$ \\
\hline$\tilde{\mathrm{D}}_{4000}$ & 0.109 & $\mathrm{Fe} 4531$ & 0.273 & $(u-g)_{\mathrm{AB}}$ & 0.0500 \\
$\mathrm{H} \delta_{\mathrm{A}}$ & 0.217 & $\mathrm{Fe} 4668$ & 0.224 & $(g-r)_{\mathrm{AB}}$ & 0.0421 \\
$\mathrm{H} \delta_{\mathrm{F}}$ & 0.281 & $\mathrm{H} \beta$ & 0.276 & $(g-i)_{\mathrm{AB}}$ & 0.0412 \\
$\mathrm{CN}_{1}$ & 0.224 & $\mathrm{Fe} 5015$ & 0.234 & $(g-z)_{\mathrm{AB}}$ & 0.0393 \\
$\mathrm{CN}_{2}$ & 0.269 & $\mathrm{Mg}_{1}$ & 0.166 & $(g-J)_{\mathrm{AB}}$ & 0.0368 \\
$\mathrm{Ca} 4227$ & 0.400 & $\mathrm{Mg}_{2}$ & 0.193 & $(g-H)_{\mathrm{AB}}$ & 0.0354 \\
$\mathrm{G} 4300$ & 0.265 & $\mathrm{Mgb}$ & 0.268 & $(g-K \mathrm{~s})_{\mathrm{AB}}$ & 0.0348 \\
$\mathrm{H} \gamma_{\mathrm{A}}$ & 0.204 & $\mathrm{Fe} 5270$ & 0.251 & $(U-B)$ & 0.0502 \\
$\mathrm{H} \gamma_{\mathrm{F}}$ & 0.274 & $\mathrm{Fe} 5335$ & 0.292 & $(B-V)$ & 0.0434 \\
$\mathrm{H} \gamma_{\mathrm{HR}}$ & 0.887 & $\mathrm{Fe} 5406$ & 0.314 & $(V-R)$ & 0.0373 \\
$\mathrm{H} \gamma_{\mathrm{VA}, 125}$ & 0.260 & $\mathrm{Fe} 5709$ & 0.291 & $(V-I)$ & 0.0367 \\
$\mathrm{H} \gamma_{\mathrm{VA}, 200}$ & 0.233 & $\mathrm{Fe} 5782$ & 0.333 & $(V-J)$ & 0.0391 \\
$\mathrm{H} \gamma_{\mathrm{VA}, 275}$ & 0.218 & $\mathrm{Na} 5895$ & 0.271 & $(V-K)$ & 0.0352 \\
$\mathrm{Fe} 4383$ & 0.280 & $\mathrm{TiO} O_{1}$ & 0.182 & $(R-I)$ & 0.0285 \\
$\mathrm{Ca} 4455$ & 0.340 & $\mathrm{TiO}_{2}$ & 0.157 & $(J-H)$ & 0.0309 \\
& & & & $(H-K)$ & 0.0257 \\
\hline
\end{tabular}

useful to measure this kind of spectral feature in logarithmic units, i.e.

$\tilde{\mathrm{D}}_{4000} \equiv 2.5 \log _{10}\left(\mathrm{D}_{4000}\right)$,

since, with this definition,

$\sigma\left(\tilde{\mathrm{D}}_{4000}\right) \simeq \frac{2.5 \log _{10} e}{10} \frac{1}{S / N(\AA)} \equiv \frac{c\left(\tilde{\mathrm{D}}_{4000}\right)}{S / N(\AA)}$,

which has the same functional form that Eq. (3). However, since the definition of this index covers a relatively large wavelength range, the computation of the averaged signal-to-noise ratio in the considered bandpasses may introduce a systematic bias in the estimation of the random error. This point is treated in more detail in Appendix A. In addition, in Appendix B we show that Eq. (3) also holds when using colors.

In summary, errors in typical spectroscopic measurements (line-strength indices and colors) can be accurately estimated as a constant divided by an appropriate average of the signal-tonoise ratio per $\AA$. Table 1 lists those constants for common linestrength features and colors. Note that for colors we have employed Eq. (B.16). For classical molecular indices (and atomic indices measured in magnitudes), which are defined with the help of three bandpasses, the error coefficients are computed as (see Eqs. (44) and (45) in Cardiel et al. 1998)

$$
\begin{aligned}
c(M)=2.5 & \log _{10}(e) \\
& \times \sqrt{\frac{1}{\Delta \lambda_{\mathrm{c}}}+\left(\frac{\lambda_{\mathrm{r}}-\lambda_{\mathrm{c}}}{\lambda_{\mathrm{r}}-\lambda_{\mathrm{b}}}\right)^{2} \frac{1}{\Delta \lambda_{\mathrm{b}}}+\left(\frac{\lambda_{\mathrm{c}}-\lambda_{\mathrm{b}}}{\lambda_{\mathrm{r}}-\lambda_{\mathrm{b}}}\right)^{2} \frac{1}{\Delta \lambda_{\mathrm{r}}}}
\end{aligned}
$$

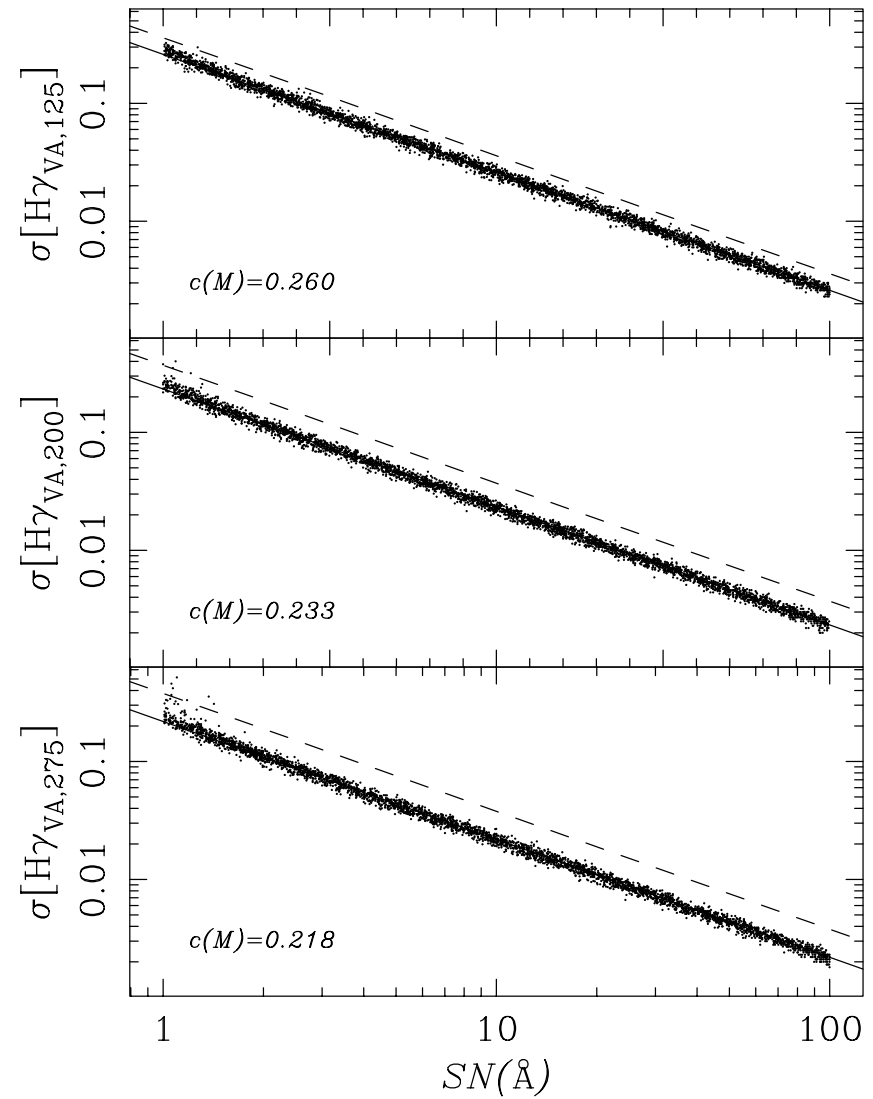

Fig. 1. Random errors from numerical simulations in the measurement of the $\mathrm{H} \gamma_{\mathrm{VA}, \sigma}$ indices defined by Vazdekis \& Arimoto (1999), as a function of the mean signal-to-noise ratio per $\AA$. The fit to the data points (full line) provides the error coefficients $c(M)$ defined in Eq. (3). The dashed line indicates what should be the location of the data if the bandpasses defining those indices did not overlap.

where $\lambda_{\mathrm{b}}, \lambda_{\mathrm{c}}$ and $\lambda_{\mathrm{r}}$ are the central wavelengths of the blue, central and red bandpasses, respectively, and $\Delta \lambda_{\mathrm{b}}, \Delta \lambda_{\mathrm{c}}$ and $\Delta \lambda_{\mathrm{r}}$ are the bandpass widths ${ }^{1}$.

It is important to highlight that the coefficients $c(M)$ obtained with the previous expression are valid as long as the three bandpasses do not overlap. If this is not the case, the coefficients can be computed numerically through numerical simulations. This is in fact the situation for the three narrow $H \gamma_{\mathrm{VA}, \sigma}$ indices introduced by Vazdekis \& Arimoto (1999), in which part of the central bandpass containing the spectral feature overlaps with the blue bandpass of the pseudo-continuum. In Fig. 1 we show the result of simulating 4000 spectra using Eq. (2), from which we have derived the $c(M)$ coefficients for $\mathrm{H} \gamma_{\mathrm{VA}, \sigma}$ by fitting the measured random errors as a function of $S / N(\AA)$.

\footnotetext{
${ }^{1}$ For those readers interested in computing expected errors in atomic indices measured in $\AA$, it is possible to use

$\sigma\left(I_{\mathrm{a}}\right) \simeq \frac{c_{1}-c_{2} I_{\mathrm{a}}}{S / N(\AA)}$

where $c_{1}$ and $c_{2}$ coefficients for typical line-strengh features are given in Table 1 of Cardiel et al. (1998). Note that in this case the error estimates depend on the absolute index value.
} 


\section{Reliability of physical properties derived from spectroscopic data}

Given an $n$-dimensional observational space, built with the help of $n$ spectroscopic measurements $M_{i}$, with $i=1, \ldots, n$, small variations in all those measurements around a given point $\left(m_{1}^{0}, m_{2}^{0}, \ldots, m_{i}^{0}, \ldots, m_{n}^{0}\right)$ in that space can be expressed, using the prediction of evolutionary synthesis models, as a linear function of $n$ variations of physical parameters $P_{j}$ around the point $\left(p_{1}^{0}, p_{2}^{0}, \ldots, p_{j}^{0}, \ldots, p_{n}^{0}\right)$ of the form

$\Delta m_{i} \simeq \sum_{j=1}^{n} a_{i j} \Delta p_{j}$

being $\left(p_{1}^{0}, p_{2}^{0}, \ldots, p_{j}^{0}, \ldots, p_{n}^{0}\right)$ the physical parameters associated to the observables $\left(m_{1}^{0}, m_{2}^{0}, \ldots, m_{i}^{0}, \ldots, m_{n}^{0}\right)$, and where in general the $a_{i j}$ coefficients are functions of $\left(m_{1}^{0}, m_{2}^{0}, \ldots, m_{i}^{0}, \ldots, m_{n}^{0}\right)$. If the $n \times n$ matrix $A$ of the coefficients $a_{i j}$ is not singular (i.e. $\operatorname{det}(A) \neq 0$ ), we can also express locally variations in the physical parameters as a function of variations in the spectroscopic measurements by

$\Delta p_{j} \simeq \sum_{i=1}^{n} b_{j i} \Delta m_{i}$

Since each set of spectroscopic measurements will be, in practice, affected by random errors $\sigma\left(m_{i}\right)$ (assumed to follow a normal distribution), a random sampling of a given astronomical object provides a cloud of points of coordinates $\left(m_{1}, m_{2}, \ldots, m_{i}, \ldots, m_{n}\right)_{k}$ (with $\left.k=1, \ldots, N_{\text {points }}\right)$ scattered around $\left(m_{1}^{0}, m_{2}^{0}, \ldots, m_{i}^{0}, \ldots, m_{n}^{0}\right)$ and following a multivariate normal distribution. Assuming that the bandpasses defining $m_{i}$ and $m_{j}$ do not overlap $\forall i, j$ with $i \neq j$ (i.e. their random errors are uncorrelated), the surfaces of constant probability density are hyperellipsoids centered at $\left(m_{1}^{0}, m_{2}^{0}, \ldots, m_{i}^{0}, \ldots, m_{n}^{0}\right)$, with axes parallel to the coordinate axes. The volume $V_{M}$ of the hyperellipsoids (for a fixed confidence level $1-\alpha$ ) in the $n$-dimensional space defined by the spectroscopic measurements will be proportional to the product of the semiaxes (Kendall 1961)

$V_{M}=\frac{2\left[\pi \chi_{n}^{2}(\alpha)\right]^{n / 2}}{n \Gamma(n / 2)} \prod_{i=1}^{n} \sigma\left(m_{i}\right)$,

where $\chi_{n}^{2}(\alpha)$ is the upper $(100 \alpha)$ th percentile of a chi-square distribution with $n$ degrees of freedom, and $\Gamma(n / 2)$ is the complete gamma function. When considering the $n$-dimensional space defined by the physical parameters $P_{j}$, the above hyperellipsoids are transformed into new objects, which in general will be no longer hyperellipsoids. However, the volume contained within these $n$-dimensional objects can still be easily computed as

$V_{P}=|\operatorname{det}(B)| V_{M}$,

where $B$ is the $n \times n$ matrix of the coefficients $b_{j i}$. In fact, replacing $\Delta p_{j}$ and $\Delta m_{i}$ in Eq. (9) by the errors $\sigma\left(p_{j}\right)$ and $\sigma\left(m_{i}\right)$ respectively, and using the result given in Eq. (3), the volume $V_{P}$ can be obtained as

$$
\begin{aligned}
V_{P} & =|\operatorname{det}(B)| \frac{2\left[\pi \chi_{n}^{2}(\alpha)\right]^{n / 2}}{n \Gamma(n / 2)} \prod_{i=1}^{n} \frac{c\left(m_{i}\right)}{S / N(\AA)_{i}} \\
& =\kappa \phi(\alpha, n) \prod_{i=1}^{n} \frac{1}{S / N(\AA)_{i}},
\end{aligned}
$$

where

$\kappa \equiv|\operatorname{det}(B)| \prod_{i=1}^{n} c\left(m_{i}\right)=\frac{1}{|\operatorname{det}(A)|} \prod_{i=1}^{n} c\left(m_{i}\right)$,

and

$\phi(\alpha, n) \equiv \frac{2\left[\pi \chi_{n}^{2}(\alpha)\right]^{n / 2}}{n \Gamma(n / 2)}$.

Summarizing, for a fixed number of spectroscopic measurements, the $\phi(\alpha, n)$ factor can be considered as a constant, and the volume interior to the surfaces of constant probability in the $n$-dimensional space constructed by the physical parameters $P_{j}$ $(j=1, \ldots, n)$, can be expressed as

$V_{P} \propto \kappa \prod_{i=1}^{n} \frac{1}{S / N(\AA)_{i}}$,

where $\kappa$ encapsulates both the sensitivity of the spectroscopic indices to the signal-to-noise ratio - through the $c\left(m_{i}\right)$ coefficients - , and the degree of degeneracy between the derived physical parameters - by way of the $a_{i j}$ or $b_{j i}$ coefficients -. For that reason $\kappa$ is a parameter that provides quantitative information concerning the suitability of a given set of spectroscopic indices to the study of the corresponding physical parameters. For our purposes, the best observational space will be that for which this suitability parameter attains the lowest value (i.e. minimum $V_{P}$ volume). In this sense, an almost orthogonal parameter space greatly helps to reduce this number, although its precise value will also be strongly constrained by the signal-tonoise dependence of the measured spectroscopic features.

\section{Age-metallicity degeneracy: A case study}

As an illustrative example, we can analyze the typical problem of breaking the age-metallicity degeneracy from twodimensional diagrams built with line-strength indices and colors.

\subsection{Computing the suitability parameter}

In this case, the observational space is defined by two spectroscopic measurements $M_{1}$ and $M_{2}$. Small variations in both indices around a given point $\left(m_{1}^{0}, m_{2}^{0}\right)$ can be expressed as a linear function of age and metallicity of the form

$\left(\begin{array}{l}\Delta m_{1} \\ \Delta m_{2}\end{array}\right) \simeq\left(\begin{array}{ll}a_{11} & a_{12} \\ a_{21} & a_{22}\end{array}\right)\left(\begin{array}{c}\Delta \log (Z) \\ \Delta \log (\text { age })\end{array}\right)$ 
where, in general, the $a_{i j}$ coefficients are functions of $m_{1}^{0}$ and $m_{2}^{0}$. It is straightforward to show that

$a_{11} \simeq S_{M_{1}} a_{12}$, and

$a_{22} \simeq \frac{a_{21}}{S_{M_{2}}}$

being $S_{M_{l}}$ the metal sensitivity parameter introduced by Worthey (1994) for the $l$ th line-strength index $M_{l}$. It is important to note that the method followed by Worthey (1994, see his footnote number 4 ) is a good approximation for the derivatives $\mathrm{d} \log ($ age $) / \mathrm{d} \log (Z)$ as long as the approximation that the $a_{i j}$ coefficients are constant in the region where age and metallicity are being measured holds. However, since this is not always the case, in Appendix $\mathrm{C}$ we describe the use of bivariate polynomials to express locally the $a_{i j}$ coefficients as a function of age and metallicity, which in turn can be employed to derive more accurate local metal sensitivity parameters through the relations given in Eq. (17).

If the $2 \times 2$ matrix $A$ of coefficients $a_{i j}$ in Eq. (16) is invertible, one can also express locally age and metallicity variations as a function of variations in the line-strength indices by

$\left(\begin{array}{c}\Delta \log (Z) \\ \Delta \log (\text { age })\end{array}\right)=\left(\begin{array}{ll}b_{11} & b_{12} \\ b_{21} & b_{22}\end{array}\right)\left(\begin{array}{l}\Delta m_{1} \\ \Delta m_{2}\end{array}\right)$,

where

$b_{11}=a_{22} / \operatorname{det}(A)$,

$b_{12}=-a_{12} / \operatorname{det}(A)$,

$b_{21}=-a_{21} / \operatorname{det}(A)$,

$b_{22}=a_{11} / \operatorname{det}(A)$.

Assuming that the indices $M_{1}$ and $M_{2}$ are independent, replacing $\Delta m_{l}$ in Eq. (18) by the random error $\sigma\left(m_{l}\right)$, and using the result given in Eq. (3), the area of the 2-dimensional errorellipse in the space defined by the physical parameters $\log (Z)$ and $\log ($ age $)$, can be computed as

$$
\begin{aligned}
V_{\log (Z), \log (\text { age })} & =\pi \chi_{2}^{2}(\alpha)|\operatorname{det}(B)| \frac{c\left(m_{1}\right)}{S / N(\AA)_{1}} \frac{c\left(m_{2}\right)}{S / N(\AA)_{2}} \\
& =\pi \chi_{2}^{2}(\alpha) \frac{1}{|\operatorname{det}(A)|} \frac{c\left(m_{1}\right)}{S / N(\AA)_{1}} \frac{c\left(m_{2}\right)}{S / N(\AA)_{2}},
\end{aligned}
$$

from which it is evident that the suitability parameter is defined as

$$
\kappa \equiv|\operatorname{det}(B)| c\left(m_{1}\right) c\left(m_{2}\right)=\underbrace{\frac{1}{|\operatorname{det}(A)|}}_{\begin{array}{c}
\text { sensitivity to } \\
\text { age-metallicity } \\
\text { degeneracy }
\end{array}} \underbrace{c\left(m_{1}\right) c\left(m_{2}\right)}_{\begin{array}{c}
\text { sensitivity } \\
\text { to } S / N(\AA)
\end{array}},
$$

where the effect of the degeneracy of the iso-metallicity and iso-age model predictions are encapsulated in the geometric transformation represented by the $a_{i j}$ coefficients (or by the $b_{i j}$ coefficients of the inverse transformation), whereas the sensitivity to $S / N(\AA)$ is controlled by the $c\left(m_{l}\right)$ factors.

Thus, it is clear that the goal of achieving minimum total errors in both age and metallicity involve the balancing of the two effects. In fact, degeneracy and sensitivity to errors typically behave in opposite senses as a function of the wavelength coverage of the considered spectroscopic feature. Narrow linestrength indices can be found to be sensitive almost only to age or to metallicity, whereas broad band features are less sensitive to noise, as can be seen in the functional dependence of $c\left(m_{l}\right)$ on bandpass widths (Eqs. (7) and (B.16) for line-strength indices and colors, respectively).

In Tables 2-4 we present suitability parameters (more precisely $\log [\kappa])$ computed for different combinations of common line-strength indices and colors. In their computation we have employed the predictions of simple stellar populations from Bruzual \& Charlot $(2001)^{2}$. In addition, in Table 5 we have also determined the suitability parameter for those combinations of line-strength indices including narrow features, that so far have been considered to provide a better discrimination between age and metallicity: Fe4668 as metallicity indicator, and $\mathrm{H} \gamma_{\mathrm{HR}}$ (Jones \& Worthey 1995), $\mathrm{H} \delta_{\mathrm{A}}, \mathrm{H} \delta_{\mathrm{F}}, \mathrm{H} \gamma_{\mathrm{A}}$ and $\mathrm{H} \gamma_{\mathrm{F}}$ (Worthey \& Ottaviani 1997), and $\mathrm{H} \gamma_{\mathrm{VA}, \sigma}$ (Vazdekis \& Arimoto 1999) as age indicators ${ }^{3}$.

\subsection{Two examples}

In Fig. 2, panels a and c, we show two examples of typical index-index diagrams. In both cases we have simulated the effect of observing a hypothetical simple stellar population $12 \mathrm{Gyr}$ old and with $[\mathrm{Fe} / \mathrm{H}]=0.093$ (corresponding to the physical parameters of one of the points predicted by the grid of models), with a signal-to-noise ratio per $\AA$ of 100 . The simulations are displayed as clouds of points clustered around the model prediction for those physical parameters. Each simulated point in the index-index diagrams has been transformed into age and metallicity using a $N=2$ bivariate polynomial transformation, as explained in Appendix C (see Fig. C.1). As a guide to the reader, we give in Table 6 the numbers involved in the computation of the suitability parameter in the two indexindex diagrams shown in Fig. 2. The difference between the physical parameters derived from each simulated point, relative to the values corresponding to the hypothetical stellar population, are represented as errors in $\log ($ age $)$ and $\log (Z)$ in panels $b$ and $d$. The ellipses show the regions of 68.26, 95.44 and $99.73 \%$ probability. The areas covered by these ellipses in the error space are clearly larger in the $\mathrm{H} \beta-\mathrm{Fe} 4668$ diagram than in the $\tilde{\mathrm{D}}_{4000}-\mathrm{Fe} 4668$ diagram (note that the axis scales in panels $b$ and $d$ are different). This result agrees with the larger (and thus worse) value of $\log [\kappa]$ for the first index-index diagram. Note, however, that the errors are more correlated in the second diagram. In fact, the standard deviations around each physical parameter, displayed with thick error bars, are a relatively fair representation of the $1 \sigma$ error ellipse in panel $\mathrm{b}$, but not in

\footnotetext{
2 Available at http://www.sdss.mpg.de/sdssMPA/ Spectral_Tools/

${ }^{3}$ In the computation of the numbers listed in Tables 2-7 we have assumed that all the spectral data are not correlated. Since some of the line-strength indices and colors overlap, we are implicitly assuming that overlapping features are obtained in independent measurements (i.e. different spectra and photometric colors).
} 
Table 2. Logarithm of the suitability index, $\log [\kappa],-$ see Eq. (21) - for the study of the age-metallicity degeneracy, computed for different combinations of common line-strength indices in the optical range. For the generation of these numbers we have employed the predictions of Bruzual \& Charlot (2001) models, for single stellar populations of 12 Gyr old and solar metallicity. Better diagrams are those for which $\kappa$ are lower. In this sense, and as a guide for the eye, we have boldfaced and underlined the $10 \%$ of these numbers with the lowest values.

\begin{tabular}{|c|c|c|c|c|c|c|c|c|c|c|c|c|c|c|c|c|c|c|c|c|c|c|}
\hline & 条 & $\bar{u}$ & 己 & $\begin{array}{l}\text { N } \\
\text { İ } \\
\text { U }\end{array}$ & 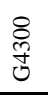 & 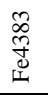 & \begin{tabular}{l}
$\stackrel{n}{f}$ \\
\multirow{z}{*}{}
\end{tabular} & $\begin{array}{l}\bar{n} \\
\text { f } \\
\text { w }\end{array}$ & 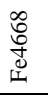 & 胥 & $\begin{array}{l}n \\
0 \\
0 \\
0 \\
0 \\
0\end{array}$ & $\sum^{500}$ & $\sum$ & ${ }^{\circ 0}$ & 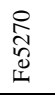 & 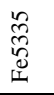 & $\begin{array}{l}0 \\
0 \\
10 \\
10 \\
1\end{array}$ & $\begin{array}{l}\stackrel{2}{2} \\
\text { in } \\
\text { I }\end{array}$ & $\begin{array}{l}0 \\
\infty \\
i n \\
0 \\
\end{array}$ & $\begin{array}{l}\tilde{} \\
\infty \\
\approx \\
z\end{array}$ & $\stackrel{\odot}{\ominus}$ & 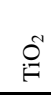 \\
\hline$\tilde{\mathrm{D}}_{4000}$ & $\ldots$ & $\underline{0.2}$ & $\underline{0.4}$ & 1.2 & 1.9 & $\underline{0.3}$ & $\underline{0.6}$ & $\underline{0.8}$ & $\underline{0.0}$ & $\underline{0.5}$ & $\underline{0.4}$ & $\underline{0.2}$ & $\underline{0.0}$ & $\underline{0.5}$ & $\underline{0.6}$ & $\underline{0.4}$ & $\underline{0.5}$ & $\underline{0.6}$ & $\underline{0.7}$ & $\underline{0.3}$ & 1.4 & $\underline{0.9}$ \\
\hline $\mathrm{CN}_{1}$ & $\cdots$ & $\ldots$ & 1.7 & 1.5 & 1.5 & 1.5 & 1.8 & 2.4 & $\underline{0.9}$ & 1.2 & 1.4 & 2.8 & 1.9 & 2.5 & 1.9 & 1.5 & 1.6 & 1.5 & 1.6 & 1.6 & 1.7 & 1.6 \\
\hline $\mathrm{CN}_{2}$ & $\ldots$ & $\ldots$ & $\ldots$ & 1.7 & 1.6 & 1.4 & 1.7 & 2.1 & $\underline{0.9}$ & 1.2 & 1.4 & 1.7 & 1.4 & 1.9 & 1.7 & 1.4 & 1.5 & 1.5 & 1.6 & 1.5 & 1.7 & 1.8 \\
\hline Ca4227 & $\ldots$ & $\ldots$ & $\ldots$ & $\ldots$ & 2.4 & 1.5 & 1.8 & 2.0 & 1.2 & 1.6 & 1.6 & 1.5 & 1.3 & 1.8 & 1.8 & 1.6 & 1.7 & 1.7 & 1.8 & 1.5 & 2.3 & 2.3 \\
\hline G4300 & $\ldots$ & $\ldots$ & $\ldots$ & $\ldots$ & $\ldots$ & 1.6 & 1.9 & 2.1 & 1.3 & 1.9 & 1.7 & 1.5 & 1.3 & 1.8 & 1.9 & 1.7 & 1.8 & 1.9 & 2.0 & 1.6 & 2.8 & 2.1 \\
\hline Fe 4383 & $\cdots$ & $\ldots$ & $\cdots$ & $\ldots$ & $\ldots$ & $\ldots$ & 3.3 & 2.3 & 1.4 & 1.4 & 1.9 & 1.5 & 1.4 & 1.8 & 2.8 & 2.0 & 2.3 & 1.9 & 2.1 & 2.1 & 1.8 & 1.5 \\
\hline Ca4455 & $\ldots$ & $\ldots$ & $\ldots$ & $\ldots$ & $\ldots$ & $\ldots$ & $\ldots$ & 2.6 & 1.7 & 1.7 & 2.2 & 1.8 & 1.7 & 2.2 & 3.3 & 2.4 & 2.6 & 2.2 & 2.4 & 2.5 & 2.1 & 1.9 \\
\hline Fe4531 & $\cdots$ & $\ldots$ & $\ldots$ & $\ldots$ & $\ldots$ & $\ldots$ & $\cdots$ & $\ldots$ & 1.6 & 1.8 & 2.1 & 2.3 & 2.5 & 2.9 & 2.7 & 2.2 & 2.3 & 2.2 & 2.3 & 2.6 & 2.2 & 2.1 \\
\hline Fe4668 & $\cdots$ & $\ldots$ & $\cdots$ & $\cdots$ & $\ldots$ & $\ldots$ & $\ldots$ & $\ldots$ & $\ldots$ & 1.2 & 2.4 & $\underline{0.9}$ & $\underline{0.8}$ & 1.3 & 1.6 & 1.9 & 1.8 & 2.3 & 3.1 & 1.2 & 1.5 & 1.2 \\
\hline $\mathrm{H} \beta$ & $\ldots$ & $\ldots$ & $\ldots$ & $\ldots$ & $\ldots$ & & $\ldots$ & $\ldots$ & $\ldots$ & $\ldots$ & 1.7 & 1.2 & $\underline{1.0}$ & 1.5 & 1.7 & 1.6 & 1.7 & 1.8 & 1.9 & 1.3 & 2.2 & 1.6 \\
\hline Fe5015 & $\ldots$ & $\ldots$ & $\ldots$ & $\cdots$ & $\ldots$ & $\ldots$ & $\cdots$ & $\ldots$ & $\ldots$ & $\cdots$ & $\cdots$ & 1.4 & 1.3 & 1.7 & 2.1 & 2.5 & 2.4 & 2.5 & 3.4 & 1.7 & 1.9 & 1.6 \\
\hline $\mathrm{Mg}_{1}$ & $\ldots$ & $\cdots$ & $\cdots$ & $\ldots$ & $\cdots$ & $\ldots$ & $\ldots$ & $\cdots$ & $\cdots$ & $\cdots$ & $\ldots$ & $\ldots$ & 1.8 & 2.4 & 1.8 & 1.5 & 1.6 & 1.5 & 1.6 & 1.6 & 1.6 & 1.6 \\
\hline $\mathrm{Mg}_{2}$ & $\ldots$ & $\ldots$ & $\ldots$ & $\ldots$ & $\cdots$ & $\ldots$ & $\ldots$ & $\ldots$ & $\cdots$ & $\ldots$ & $\ldots$ & $\ldots$ & $\cdots$ & 2.8 & 1.8 & 1.3 & 1.5 & 1.3 & 1.5 & 1.6 & 1.5 & 1.4 \\
\hline $\mathrm{Mgb}$ & $\cdots$ & $\ldots$ & $\cdots$ & $\ldots$ & $\cdots$ & $\ldots$ & $\ldots$ & $\ldots$ & $\cdots$ & $\cdots$ & $\ldots$ & $\ldots$ & $\ldots$ & $\cdots$ & 2.3 & 1.8 & 1.9 & 1.8 & 2.0 & 2.0 & 2.0 & 1.9 \\
\hline Fe5270 & $\ldots$ & $\ldots$ & $\cdots$ & $\ldots$ & $\ldots$ & $\ldots$ & $\ldots$ & $\ldots$ & $\ldots$ & $\ldots$ & $\ldots$ & $\ldots$ & $\ldots$ & $\ldots$ & $\ldots$ & 2.3 & 2.5 & 2.1 & 2.3 & 2.7 & 2.1 & 1.9 \\
\hline Fe5335 & $\cdots$ & $\ldots$ & $\cdots$ & $\cdots$ & $\cdots$ & $\ldots$ & $\cdots$ & $\cdots$ & $\cdots$ & $\cdots$ & $\cdots$ & $\cdots$ & $\cdots$ & $\cdots$ & $\ldots$ & $\cdots$ & 2.8 & 2.3 & 2.6 & 1.8 & 1.9 & 1.6 \\
\hline Fe5406 & $\ldots$ & $\ldots$ & $\ldots$ & $\ldots$ & $\cdots$ & $\ldots$ & $\cdots$ & $\ldots$ & $\ldots$ & $\cdots$ & $\cdots$ & $\cdots$ & $\ldots$ & $\cdots$ & $\ldots$ & $\ldots$ & $\cdots$ & 2.3 & 2.6 & 2.0 & 2.0 & 1.7 \\
\hline Fe5709 & & $\cdots$ & $\ldots$ & $\cdots$ & $\cdots$ & $\cdots$ & $\cdots$ & $\cdots$ & $\cdots$ & $\ldots$ & $\cdots$ & $\cdots$ & $\ldots$ & $\ldots$ & $\ldots$ & $\ldots$ & $\ldots$ & & 2.9 & 1.7 & 2.1 & 1.7 \\
\hline Fe5782 & $\ldots$ & $\ldots$ & $\cdots$ & $\ldots$ & $\ldots$ & $\ldots$ & $\cdots$ & $\ldots$ & $\cdots$ & $\cdots$ & $\cdots$ & $\ldots$ & $\ldots$ & $\cdots$ & $\cdots$ & $\cdots$ & $\cdots$ & $\ldots$ & $\ldots$ & 1.9 & 2.2 & 1.8 \\
\hline Na5895 & $\ldots$ & $\ldots$ & $\ldots$ & $\ldots$ & $\ldots$ & $\ldots$ & $\ldots$ & $\ldots$ & $\cdots$ & $\ldots$ & $\ldots$ & $\ldots$ & $\ldots$ & $\ldots$ & $\ldots$ & $\ldots$ & $\cdots$ & $\ldots$ & $\ldots$ & $\ldots$ & 1.8 & 1.6 \\
\hline $\mathrm{TiO}_{1}$ & $\cdots$ & $\ldots$ & $\cdots$ & $\ldots$ & $\cdots$ & $\ldots$ & $\ldots$ & $\ldots$ & $\cdots$ & $\ldots$ & $\ldots$ & $\cdots$ & $\cdots$ & $\ldots$ & $\cdots$ & $\cdots$ & $\cdots$ & $\cdots$ & $\cdots$ & $\cdots$ & $\cdots$ & 2.2 \\
\hline $\mathrm{TiO}_{2}$ & $\cdots$ & $\ldots$ & $\ldots$ & $\ldots$ & $\ldots$ & 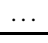 & $\ldots$ & $\ldots$ & $\ldots$ & $\ldots$ & $\ldots$ & $\ldots$ & $\ldots$ & $\ldots$ & $\ldots$ & $\ldots$ & $\ldots$ & $\ldots$ & $\ldots$ & $\ldots$ & $\ldots$ & $\ldots$ \\
\hline
\end{tabular}

Table 3. Same than Table 2 but for different combinations of colors.

\begin{tabular}{|c|c|c|c|c|c|c|c|c|c|c|c|c|c|c|c|c|}
\hline & $\begin{array}{l}0 \\
\text { on } \\
1 \\
\vdots \\
\Xi\end{array}$ & $\begin{array}{l}\frac{m}{i} \hat{i} \\
i \\
0 \\
0\end{array}$ & $\begin{array}{l}\stackrel{\infty}{a} \\
\hat{i} \\
0 \\
0\end{array}$ & $\begin{array}{l}\frac{0}{4} \\
\frac{1}{1} \\
1 \\
0\end{array}$ & $\begin{array}{l}\stackrel{\infty}{*} \\
\stackrel{a}{1} \\
0 \\
0\end{array}$ & $\begin{array}{l}\infty \\
\text { In } \\
1 \\
0 \\
0\end{array}$ & 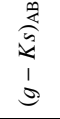 & $\begin{array}{l}\widehat{\vartheta} \\
1 \\
2\end{array}$ & $\begin{array}{l}5 \\
1 \\
0\end{array}$ & $\begin{array}{l}\approx \\
1 \\
\approx\end{array}$ & $\begin{array}{l}\overrightarrow{1} \\
1 \\
\geq\end{array}$ & $\begin{array}{l}5 \\
1 \\
\theta\end{array}$ & $\begin{array}{l}\approx \\
1 \\
\geq\end{array}$ & $\begin{array}{l}\approx \\
1 \\
\Xi\end{array}$ & $\begin{array}{l}\underset{I}{1} \\
=\end{array}$ & $\begin{array}{l}\sqrt{2} \\
1 \\
\pm\end{array}$ \\
\hline$(u-g)_{\mathrm{AB}}$ & $\ldots$ & -1.1 & -1.6 & -1.6 & -1.7 & -1.9 & -2.0 & -0.8 & -1.1 & -1.1 & -1.6 & -1.8 & -2.1 & -1.6 & $-1.1-$ & -1.7 \\
\hline$(g-r)_{\mathrm{AB}}$ & $\ldots$ & $\ldots$ & -1.0 & -0.6 & -1.6 & -1.7 & -1.9 & -1.2 & 0.7 & -0.4 & -1.1 & -1.6 & -1.9 & -1.1 & $-0.9-$ & -1.4 \\
\hline$(g-i)_{\mathrm{AB}}$ & $\ldots$ & $\ldots$ & $\ldots$ & -1.2 & -2.0 & -2.1 & -2.2 & -1.6 & -1.0 & -0.4 & -0.8 & -1.9 & -2.1 & -1.1 & $-1.1-$ & -1.6 \\
\hline$(g-z)_{\mathrm{AB}}$ & $\ldots$ & $\ldots$ & $\ldots$ & $\ldots$ & -2.0 & $\underline{-2.1}$ & $\underline{-2.2}$ & -1.6 & -0.5 & -0.4 & -1.3 & -2.0 & -2.2 & -1.4 & $-1.2-$ & -1.8 \\
\hline$(g-J)_{\mathrm{AB}}$ & $\ldots$ & $\ldots$ & $\ldots$ & $\ldots$ & $\ldots$ & -1.3 & -1.8 & -1.5 & -1.6 & -1.5 & -1.9 & -1.5 & -2.0 & -1.8 & $-1.1-$ & -1.8 \\
\hline$(g-H)_{\mathrm{AB}}$ & $\ldots$ & $\ldots$ & $\ldots$ & $\ldots$ & & $\ldots$ & -1.7 & -1.7 & -1.7 & -1.6 & $\underline{-2.0}$ & -1.4 & -2.0 & -1.9 & $-1.1-$ & -1.9 \\
\hline$(g-K s)_{\mathrm{AB}}$ & .. & $\ldots$ & $\cdots$ & $\ldots$ & $\ldots$ & $\ldots$ & $\ldots$ & -1.9 & -1.8 & -1.7 & -2.1 & -0.9 & -1.7 & -2.0 & $-1.0-$ & -1.9 \\
\hline$(U-B)$ & $\ldots$ & $\ldots$ & $\ldots$ & $\ldots$ & $\ldots$ & $\ldots$ & $\ldots$ & $\ldots$ & -1.1 & -1.1 & -1.6 & -1.6 & -1.9 & -1.5 & $-1.0-$ & -1.6 \\
\hline$(B-V)$ & $\ldots$ & $\ldots$ & $\ldots$ & $\ldots$ & $\ldots$ & $\ldots$ & $\ldots$ & $\ldots$ & $\ldots$ & -0.3 & -1.0 & -1.6 & -1.8 & -1.1 & $-0.8-$ & -1.4 \\
\hline$(V-R)$ & $\ldots$ & $\ldots$ & $\ldots$ & $\ldots$ & $\ldots$ & $\ldots$ & $\ldots$ & $\ldots$ & $\ldots$ & $\ldots$ & -0.6 & -1.5 & -1.7 & -0.8 & $-0.7-$ & -1.2 \\
\hline$(V-I)$ & $\ldots$ & $\ldots$ & $\ldots$ & $\ldots$ & $\ldots$ & $\ldots$ & $\ldots$ & $\ldots$ & $\ldots$ & $\ldots$ & $\ldots$ & -1.9 & $\underline{-2.1}$ & -0.8 & $-1.1-$ & -1.5 \\
\hline$(V-J)$ & $\ldots$ & $\ldots$ & $\ldots$ & $\ldots$ & $\ldots$ & $\ldots$ & $\ldots$ & $\ldots$ & $\ldots$ & $\ldots$ & $\ldots$ & $\ldots$ & -1.6 & -1.8 & $-0.8-$ & -1.7 \\
\hline$(V-K)$ & $\ldots$ & $\ldots$ & $\ldots$ & $\ldots$ & $\ldots$ & $\ldots$ & $\ldots$ & $\ldots$ & $\ldots$ & $\ldots$ & $\ldots$ & $\ldots$ & $\ldots$ & -1.9 & $-0.7-$ & -1.7 \\
\hline$(R-I)$ & $\ldots$ & $\ldots$ & $\ldots$ & $\ldots$ & $\ldots$ & $\ldots$ & $\ldots$ & $\ldots$ & $\ldots$ & $\ldots$ & $\ldots$ & $\ldots$ & $\ldots$ & $\ldots$ & $-0.9-$ & -1.3 \\
\hline$(J-H)$ & $\ldots$ & $\ldots$ & $\ldots$ & $\ldots$ & $\ldots$ & $\ldots$ & $\ldots$ & $\ldots$ & $\ldots$ & $\ldots$ & $\ldots$ & $\ldots$ & $\ldots$ & $\ldots$ & $\ldots-$ & -0.6 \\
\hline$(H-K)$ & $\ldots$ & $\ldots$ & $\ldots$ & $\ldots$ & $\ldots$ & $\ldots$ & $\ldots$ & $\ldots$ & $\ldots$ & $\ldots$ & $\ldots$ & $\ldots$ & $\ldots$ & $\ldots$ & $\ldots$ & $\ldots$ \\
\hline
\end{tabular}

panel d. For that reason, the standard deviations by themselves are not a good parametrization of the actual uncertainty in the derived parameters.

In practice, when one tries to answer the question of whether the integrated light of two stellar populations share the same underlying physical parameters within the error bars, the question translates into whether their error ellipses overlap in the space defined by those physical parameters. Since the probability of overlapping decreases as the area of the error ellipses becomes smaller, the suitability parameter is a direct indication of such probability. The presence of correlation between the errors is not critical, as far as the fake relationship, introduced by 
Table 4. Same than Table 2 but for different combinations of line-strength indices and colors.

\begin{tabular}{|c|c|c|c|c|c|c|c|c|c|c|c|c|c|c|c|c|c|c|c|c|c|c|}
\hline & ఫి & $\bar{u}$ & z̃ & $\begin{array}{l}\text { İ } \\
\text { İ } \\
\tilde{U}\end{array}$ & $\begin{array}{l}8 \\
\text { वे } \\
\text { Oे }\end{array}$ & $\begin{array}{l}m \\
\infty \\
\tilde{N} \\
\pm \\
I\end{array}$ & $\begin{array}{l}\text { 辇 } \\
\text { f } \\
\text { Un }\end{array}$ & \begin{tabular}{l}
$\overrightarrow{\widetilde{n}}$ \\
fo \\
\multirow{4}{*}{}
\end{tabular} & 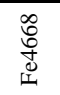 & 象 & 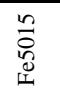 & $\sum^{\overrightarrow{50}}$ & $\sum^{\infty}$ & $\sum_{\Sigma}^{\infty}$ & 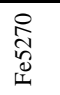 & 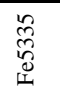 & $\begin{array}{l}0 \\
0 \\
0 \\
0 \\
\text { L }\end{array}$ & $\begin{array}{l}\stackrel{2}{0} \\
\text { in } \\
\text { i }\end{array}$ & 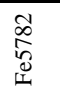 & 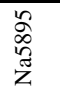 & $\stackrel{\mathcal{O}}{F}$ & $\overbrace{\mathscr{O}}^{\prime \prime}$ \\
\hline$(u-g)_{\mathrm{AB}}$ & -0.7 & -0.2 & -0.0 & 1.1 & 0.5 & -0.2 & 0.1 & $0.3=$ & -0.6 & -0.1 & -0.1 & -0.2 & -0.4 & 0.1 & 0.1 & -0.1 & -0.0 & -0.0 & 0.1 & -0.2 & 0.5 & 0.8 \\
\hline$(g-r)_{\mathrm{AB}}$ & -0.1 & -0.1 & -0.0 & 0.7 & 1.4 & -0.0 & 0.3 & 0.5 & -0.3 & 0.3 & 0.1 & -0.1 & -0.3 & 0.2 & 0.3 & 0.1 & 0.2 & 0.3 & 0.4 & -0.0 & 1.4 & 0.5 \\
\hline$(g-i)_{\mathrm{AB}}$ & -0.9 & -0.4 & -0.4 & 0.2 & 0.5 & -0.3 & 0.1 & $0.2=$ & -0.5 & 0.3 & -0.1 & -0.4 & -0.6 & -0.1 & 0.0 & -0.1 & -0.0 & 0.1 & 0.2 & -0.3 & 1.1 & 0.0 \\
\hline$(g-z)_{\mathrm{AB}}$ & -0.7 & -0.5 & -0.4 & 0.2 & 0.8 & -0.4 & -0.0 & $0.1=$ & $\underline{-0.7}$ & 0.0 & -0.2 & -0.5 & -0.7 & -0.2 & -0.1 & -0.2 & -0.1 & -0.1 & 0.0 & -0.4 & 1.4 & 0.1 \\
\hline$(g-J)_{\mathrm{AB}}$ & -1.3 & 0.4 & 0.4 & -0.0 & -0.0 & -0.2 & 0.2 & $0.6=$ & -0.7 & -0.4 & -0.2 & 0.5 & -0.0 & 0.5 & 0.2 & -0.1 & -0.0 & -0.1 & 0.0 & -0.0 & 0.1 & 0.1 \\
\hline$(g-H)_{\mathrm{AB}}$ & -1.4 & 1.1 & 0.1 & -0.1 & -0.1 & -0.1 & 0.2 & $0.8=$ & -0.7 & $\underline{-0.4}$ & -0.2 & 0.9 & 0.3 & 1.0 & 0.2 & -0.2 & -0.0 & -0.2 & -0.0 & 0.0 & & -0.0 \\
\hline$(g-K s)_{\mathrm{AB}}$ & -1.5 & -0.1 & -0.3 & -0.3 & -0.3 & 0.1 & 0.4 & $1.0=$ & $\underline{-0.7}$ & $\underline{-0.5}$ & -0.2 & -0.1 & -0.1 & 0.3 & 0.5 & -0.1 & 0.1 & -0.2 & 0.0 & 0.5 & -0.1 & -0.3 \\
\hline$(U-B)$ & -0.7 & -0.0 & 0.2 & 0.7 & 0.5 & -0.1 & 0.2 & $0.5=$ & $\underline{-0.5}$ & -0.1 & -0.0 & -0.0 & -0.3 & 0.2 & 0.2 & -0.0 & 0.1 & 0.1 & 0.2 & -0.1 & 0.5 & 2.4 \\
\hline$(B-V)$ & -0.1 & -0.1 & 0.0 & 0.7 & 1.4 & -0.0 & 0.3 & 0.5 & -0.3 & 0.3 & 0.2 & -0.1 & -0.3 & 0.2 & 0.3 & 0.2 & 0.2 & 0.3 & 0.4 & -0.0 & 1.4 & 0.5 \\
\hline$(V-R)$ & -0.3 & 0.0 & 0.1 & 0.7 & 1.1 & 0.2 & 0.5 & 0.6 & -0.1 & 0.6 & 0.3 & 0.0 & -0.2 & 0.3 & 0.5 & 0.3 & 0.4 & 0.5 & 0.6 & 0.1 & 2.1 & 0.5 \\
\hline$(V-I)$ & $\underline{-0.9}$ & -0.4 & -0.3 & 0.2 & 0.4 & -0.2 & 0.1 & $0.2=$ & -0.4 & 0.5 & -0.0 & -0.4 & -0.6 & -0.1 & 0.1 & -0.0 & 0.0 & 0.2 & 0.2 & -0.3 & 0.9 & 0.1 \\
\hline$(V-J)$ & -1.3 & 0.2 & -0.1 & -0.1 & -0.0 & 0.2 & 0.6 & $1.7=$ & $\underline{-0.5}$ & -0.3 & 0.0 & 0.2 & 0.3 & 0.7 & 0.7 & 0.1 & 0.2 & 0.1 & 0.2 & 0.5 & & -0.0 \\
\hline$(V-K)$ & -1.6 & -0.3 & $\underline{-0.5}$ & -0.3 & -0.3 & 1.2 & 3.4 & $0.5=$ & $\underline{-0.5}$ & $\underline{-0.5}$ & 0.0 & -0.4 & -0.4 & 0.0 & 1.1 & 0.2 & 0.4 & -0.0 & 0.2 & 0.3 & -0.1 & -0.3 \\
\hline$(R-I)$ & -0.9 & -0.3 & -0.2 & 0.2 & 0.4 & -0.1 & 0.2 & 0.3 & -0.3 & 1.1 & 0.1 & -0.3 & $\underline{-0.5}$ & 0.0 & 0.2 & 0.1 & 0.2 & 0.3 & 0.4 & -0.1 & 0.8 & 0.1 \\
\hline & $\underline{-0.6}$ & 0.5 & 0.4 & 0.6 & 0.7 & 1.2 & 1.5 & 1.3 & 0.7 & 0.6 & 1.3 & 0.5 & 0.4 & 0.9 & 1.4 & 1.7 & 2.5 & 1.2 & 1.4 & 0.9 & 0.9 & 0.6 \\
\hline$(H-K)$ & -1.1 & -0.2 & -0.2 & 0.0 & 0.2 & 0.1 & 0.4 & 0.4 & 0.3 & 0.2 & 0.7 & -0.3 & -0.4 & 0.1 & 0.4 & 0.5 & 0.5 & 1.3 & 1.0 & 0.0 & 0.4 & 0.0 \\
\hline
\end{tabular}

Table 5. $\mathrm{H} \gamma_{\mathrm{VA}, \sigma}$ correspond to the $[\mathrm{H} \gamma+1 / 2(\mathrm{Fe} \mathrm{I}+\mathrm{Mg} \mathrm{I})]_{\sigma}$ indices defined by Vazdekis \& Arimoto (1999). Model references are W94: Worthey (1994); JW95: Jones \& Worthey (1995); WO97: Worthey \& Ottaviani (1997); and V99: Vazdekis \& Arimoto (1999).

\begin{tabular}{ccc}
\hline \hline Index-index diagram & $\log [\kappa]$ & Models \\
\hline $\mathrm{H} \gamma_{\mathrm{HR}}$ vs. Fe4668 & 1.3 & W94,JW95 \\
$\mathrm{H} \delta_{\mathrm{A}}$ vs. Fe4668 & 0.7 & W94,WO96 \\
$\mathrm{H} \delta_{\mathrm{F}}$ vs. Fe4668 & 0.9 & W94,WO96 \\
$\mathrm{H} \gamma_{\mathrm{A}}$ vs. Fe4668 & 0.7 & W94,WO96 \\
$\mathrm{H} \gamma_{\mathrm{F}}$ vs. Fe4668 & 0.8 & W94,WO96 \\
$\mathrm{H} \gamma_{\mathrm{VA}, 125}$ vs. Fe4668 & 1.5 & VA99 \\
$\mathrm{H} \gamma_{\mathrm{VA}, 200}$ vs. Fe4668 & 1.7 & VA99 \\
$\mathrm{H} \gamma_{\mathrm{VA}, 275}$ vs. Fe4668 & 1.8 & VA99 \\
\hline
\end{tabular}

the presence of error correlation, is taken into account when studying measurements performed in different objects. In this sense, the use of numerical simulations may help to analyze the relative contribution of such error correlations to the intrinsic relationships between the physical parameters (e.g. Kuntschner et al. 2001).

\subsection{Selecting the most suitable diagram}

It is clear from the previous discussion that the best $M_{1}-M_{2}$ diagram to disentangle physical parameters (and, in particular, age and metallicity) will be that for which the factor

$\psi_{M_{1}, M_{2}}=\kappa \frac{1}{S / N(\AA)_{1}} \frac{1}{S / N(\AA)_{2}}$

is minimum. Although initially this can be achieved by selecting the combination of spectral measurements (indices and colors) for which $\log [\kappa]$ is lowest, in practice one should consider realistic signal-to-noise ratios. For that reason, and although at the light of the results displayed in Tables 2-5 it seems that color-color diagrams are the best option, in practice this is not necessarily the case. For instance, typical random errors in the measurement of colors are of the order of $0.01 \mathrm{mag}$ (e.g.
Babu \& Feigelson 1996), which implies signal-to-noise ratios per $\AA$ of the order of a few (see Fig. B.1). However, spectroscopic line-strength indices are commonly obtained with $S / N(\AA) \gtrsim 10$ times larger.

In order to compare the suitability of diagrams built with two line-strength indices, two colors, or one line-strength index and one color, let assume that

$S / N(\AA)_{\text {line-strength index }} \simeq 10 S / N(\AA)_{\text {color }}$.

Under this hypothesis, Eq. (22) provides

$\psi_{\text {color }_{1}, \text { color }_{2}} \simeq 100 \frac{\kappa_{\text {color }_{1}, \text { color }_{2}}}{\kappa_{\text {index }_{1}, \text { index }_{2}}} \psi_{\text {index }_{1}, \text {,ndex }_{2}}$,

and

$\psi_{\text {index,color }} \simeq 10 \frac{\kappa_{\text {index,color }}}{\kappa_{\text {index }_{1}, \text {,ndex }_{2}}} \psi_{\text {index }_{1}, \text {,ndex }_{2}}$.

Considering that the values displayed in Tables 2-5 correspond to $\log [\kappa]$, and focusing in the best values (the ones highlighted and underlined in these tables), we see that

$\frac{\kappa_{\text {color-color }}}{\kappa_{\text {index-index }}} \simeq 0.01$,

and

$\frac{\kappa_{\text {index }- \text { color }}}{\kappa_{\text {index-index }}} \simeq 0.1$.

Finally, combining all these numbers we obtain

$\psi_{\text {color }_{1}, \text { color }_{2}}^{\text {best }} \simeq \psi_{\text {index }_{1}, \text { color }_{2}}^{\text {best }} \simeq \psi_{\text {index }_{1}, \text { index }_{2}}^{\text {best }}$.

This result, derived after assuming the crude hypothesis stated in Eq. (23), indicates that the best index-index, index-color and color-color diagrams are, initially, almost equally suited for the study of the age-metallicity degeneracy (in 12 Gyr-old simple stellar populations with solar metallicity). In a real situation, the selection of line-strength features, colors, or a combination of both to build a good diagram will depend on the available signal-to-noise ratio for each spectral indicator. 

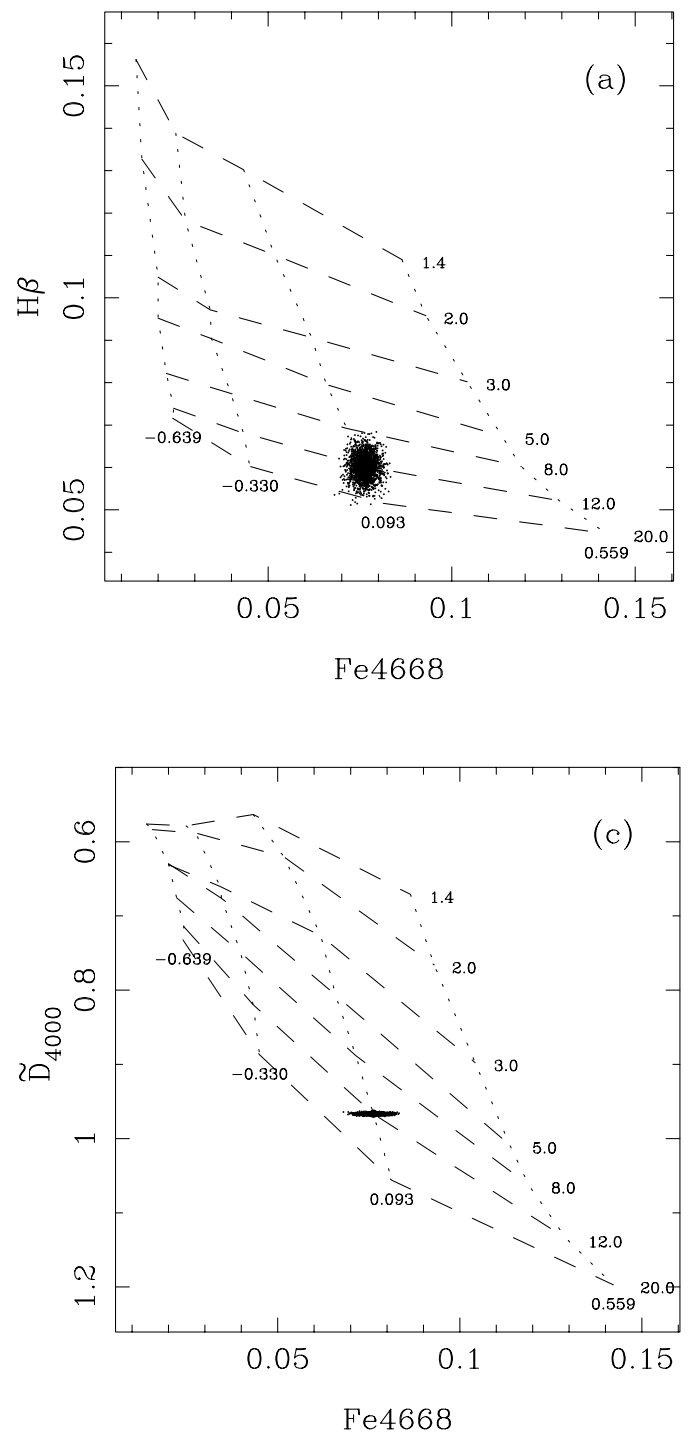
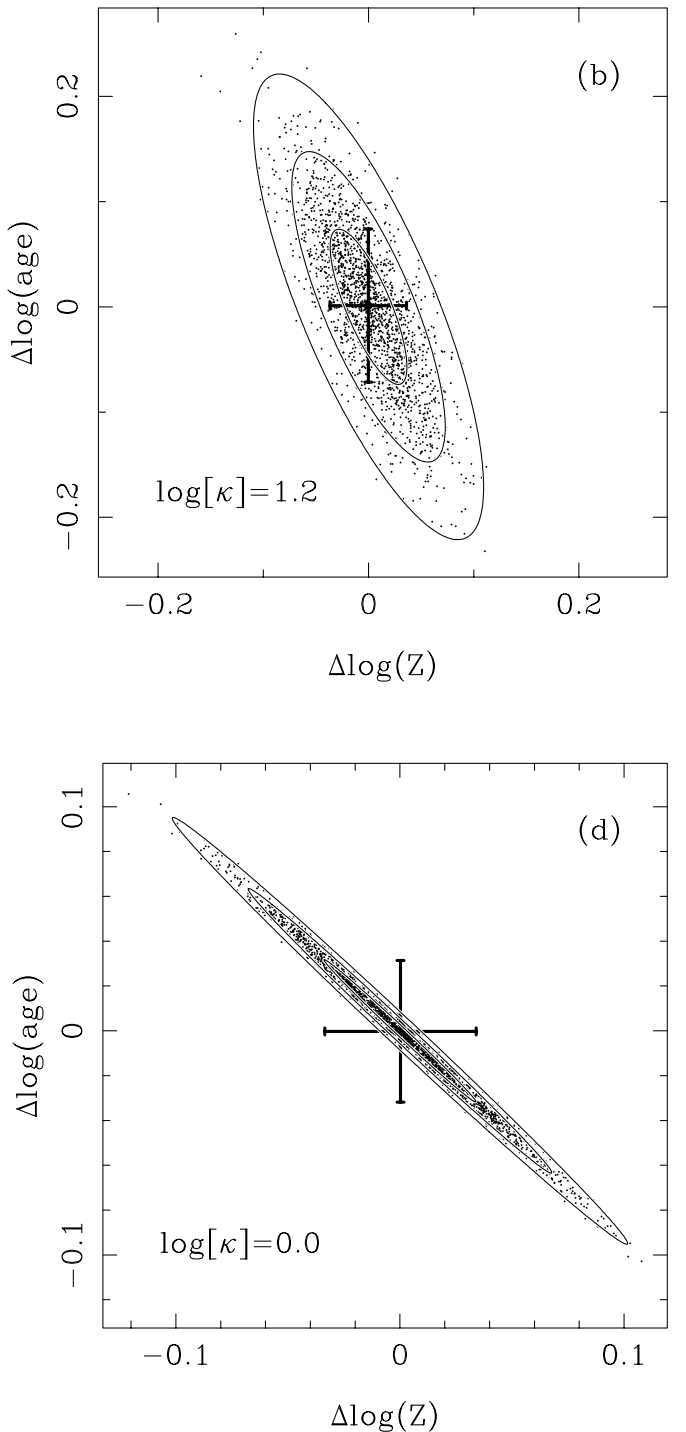

Fig. 2. Panel a): H $\beta-F e 4668$ diagram, with Bruzual \& Charlot (2001) models over-plotted (dashed lines and dotted lines correspond to the predictions for constant age and metallicity, respectively; ages are labeled in $\mathrm{Gyr}$, and metallicities are given as [Fe/H]). Dots correspond to 2000 simulations of a 12 Gyr old object with solar metallicity, assuming $S / N(\AA)=100$. Panel b): Errors in $\log ($ age $)$ and $\log (Z)$ for the same simulated data displayed in panel a). Ellipses indicate the regions of 68.26, 95.44 and $99.73 \%$ probability, whereas the central error bars show the unbiased standard deviation in each axis. Panels c) and d): Same than panels a) and b) after introducing the $\tilde{\mathrm{D}}_{4000}$ index instead of $\mathrm{H} \beta$, and using the same $S / N(\AA)$ in the simulations. The $\tilde{\mathrm{D}}_{4000}$ index is plotted in an inverse scale to display the model predictions with roughly the same orientation in age and metallicity than in panel a). Note also that the axis scales in panels b) and d) are different. See discussion in Sect. 4.2.

Focusing on the results displayed in Tables $2-4$, and leaving in a second plane the relevance of the signal-to-noise ratio just discussed, the $\tilde{\mathrm{D}}_{4000}, \mathrm{Fe} 4668$ and $\mathrm{Mg}_{2}$ features are the best line-strength features to be included in index-index and indexcolor diagrams, whereas for color-color diagrams the lowest $\log [\kappa]$ values are obtained for colors involving well separated bandpasses, like $\left(g-K_{\mathrm{s}}\right)$, and $(V-K)$.

Interestingly, the suitability parameter for combinations of narrow line-strength features with Fe4668 (displayed in Table 5) are worse than the value for the $\tilde{\mathrm{D}}_{4000}-\mathrm{Fe} 4468$ diagram, and only $\mathrm{H} \delta_{\mathrm{A}}, \mathrm{H} \delta_{\mathrm{F}}, \mathrm{H} \gamma_{\mathrm{A}}$, and $\mathrm{H} \gamma_{\mathrm{F}}$ can rival with $\mathrm{Mg}_{2}$. If, in addition, we consider that the $c(M)$ coefficients (Table 1) for the narrow indices are larger than the same coefficients for the broader features $\tilde{\mathrm{D}}_{4000}$ and $\mathrm{Mg}_{2}$, at a fixed signal-to-noise ratio per $\AA$ the diagrams of these two latest spectral features with $\mathrm{Fe} 4668$ provide more information.

\subsection{Some words of caution}

It is very important to keep in mind that, for several reasons, the above results must be taken with care:

(i) The list of line-strength indices and colors explored is, obviously, not complete.

(ii) The derived numbers do not take into account the uncertainties in the stellar population modeling. In fact, in Table 7 we compare the suitability parameters derived using the predictions of three different sets of models. The differences exhibited by some combinations of 
Table 6. Numerical values for the example of Sect. 4.2 (see also Fig. 2). The upper table lists the input line-strength indices employed in the computation of the bivariate polynomial transformations in Figs. 2a, c around the model predictions for a SSP of $12 \mathrm{Gyr}$ with solar metallicity (boldfaced). The lower table displays the different parameters involved in the calculation of the previous transformations, and the factors leading to the suitability index in Eq. (21).

\begin{tabular}{|c|c|c|c|c|}
\hline \multicolumn{5}{|c|}{ Line-strength indices from $\mathrm{BC} 01$} \\
\hline & & \multicolumn{3}{|c|}{$[\mathrm{Fe} / \mathrm{H}]$} \\
\hline & & -0.330 & 0.093 & 0.059 \\
\hline \multirow{3}{*}{$\tilde{\mathrm{D}}_{4000}$} & $8 \mathrm{Gyr}$ & 0.749313 & 0.888150 & 1.062612 \\
\hline & $12 \mathrm{Gyr}$ & 0.819419 & 0.966693 & 1.135206 \\
\hline & $20 \mathrm{Gyr}$ & 0.887191 & 1.056064 & 1.197137 \\
\hline \multirow{3}{*}{$\mathrm{H} \beta$} & $8 \mathrm{Gyr}$ & 0.077433 & 0.069191 & 0.060212 \\
\hline & $12 \mathrm{Gyr}$ & \multirow{2}{*}{0.068105} & 0.060252 & 0.052139 \\
\hline & $20 \mathrm{Gyr}$ & & 0.051545 & 0.044401 \\
\hline \multirow{3}{*}{$\mathrm{Fe} 4668$} & $8 \mathrm{Gyr}$ & 0.039794 & 0.071434 & 0.118949 \\
\hline & $12 \mathrm{Gyr}$ & \multirow{2}{*}{$\begin{array}{l}0.043234 \\
0.045004\end{array}$} & 0.076271 & 0.129336 \\
\hline & $20 \mathrm{Gyr}$ & & 0.081293 & 0.142160 \\
\hline \multirow[b]{2}{*}{ parameter } & \multicolumn{3}{|c|}{ diagram } & Eq. \\
\hline & \multicolumn{2}{|c|}{$\mathrm{H} \beta-\mathrm{Fe} 4668$} & $\tilde{\mathrm{D}}_{4000}-\mathrm{Fe} 4668$ & reference \\
\hline$p_{10}$ & \multicolumn{2}{|c|}{-0.430095} & -0.430095 & (C.2) \\
\hline$p_{11}$ & \multicolumn{2}{|c|}{0.051432} & 0.051432 & (C.2) \\
\hline$p_{20}$ & \multicolumn{2}{|c|}{0.040343} & 0.040343 & (C.2) \\
\hline$p_{01}$ & \multicolumn{2}{|c|}{0.323917} & 0.323917 & (C.2) \\
\hline$p_{02}$ & \multicolumn{2}{|c|}{-0.014719} & -0.014719 & (C.2) \\
\hline$q_{10}$ & \multicolumn{2}{|c|}{-0.058764} & 0.488931 & (C.2) \\
\hline$q_{11}$ & \multicolumn{2}{|c|}{0.003947} & -0.013260 & (C.2) \\
\hline$q_{20}$ & \multicolumn{2}{|c|}{0.002449} & -0.013883 & (C.2) \\
\hline$q_{01}$ & \multicolumn{2}{|c|}{-0.717729} & 4.921280 & (C.2) \\
\hline$q_{02}$ & \multicolumn{2}{|c|}{0.033402} & -0.225252 & (C.2) \\
\hline log[age] & \multicolumn{2}{|c|}{10.079182} & 10.079182 & (C.2) \\
\hline $\log [\mathrm{Z}]$ & \multicolumn{2}{|c|}{0.093200} & 0.093200 & (C.2) \\
\hline$a_{11}$ & \multicolumn{2}{|c|}{0.095814} & 0.095814 & $(16),(\mathrm{C} .2)$ \\
\hline$a_{12}$ & 0.03 & 31999 & 0.031999 & $(16),(\mathrm{C} .2)$ \\
\hline$a_{21}$ & -0.01 & 18528 & 0.352696 & (16), (C.2) \\
\hline$a_{22}$ & -0.04 & 44036 & 0.379328 & (16), (C.2) \\
\hline$c\left(m_{1}\right)$ & & .2757 & 0.1086 & $(21)$ \\
\hline$c\left(m_{2}\right)$ & & .2235 & 0.2235 & (21) \\
\hline$\kappa$ & & 6.995 & 0.9685 & (21) \\
\hline
\end{tabular}

line-strength indices reflect the existing discrepancies between different models. However, it is worth noting that for those combinations involving Fe4668 the agreement is very reasonable, and less good for $\tilde{\mathrm{D}}_{4000}, \mathrm{Mg}_{2}$, and Na5895.

(iii) The integrated light of the stellar systems under study (e.g. star clusters, galaxies) are not necessarily well described by simple stellar populations. New models, including more complex star formation histories, may provide a different suitability ranking of line-strength features and colors. Anyway, the procedure here described is still valid as long as the correct models are employed.

(iv) When comparing several objects in a diagram, their physical parameters are expected to exhibit a range. Since the $\kappa$ values shown in Tables $2-5$ have been computed for a fixed age and metallicity, the suitability parameter will be different for each object. For illustration, in Fig. 3 we

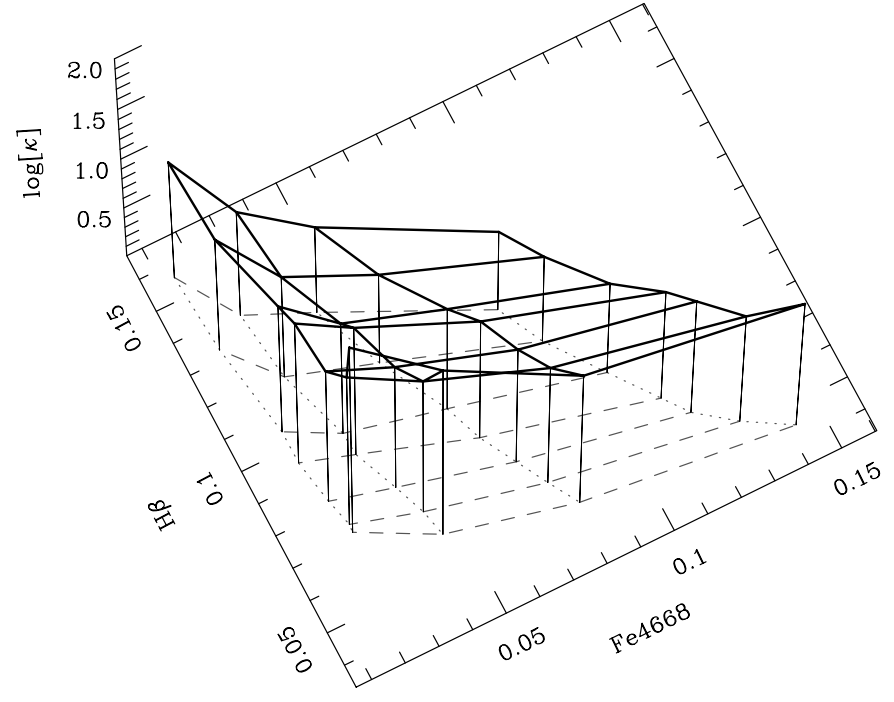

Fig. 3. Three-dimensional representation of the $\log [\kappa]$ values for all the intersecting points in the grid of models from Bruzual \& Charlot (2001), for the $\mathrm{H} \beta-\mathrm{Fe} 4668$ diagram.

represent a three-dimensional plot with the $\log [\kappa]$ values derived for each intersecting point constituting the grid of models in the $\mathrm{H} \beta-\mathrm{Fe} 4668$ diagram. It is clear from this figure that the suitability parameter is a function of the location in the index-index plane.

(v) Although systematic effects have not been considered in the analysis performed so far (see Sect. 2.1), in the real world they can be the ones that, if not properly constrained, may determine the actual suitability of a given set of spectroscopic observables. In practice the nature and relevance of systematic errors affecting line-strength indices (e.g. spectral resolution, radial velocity, flux calibration) and colors (e.g. zeropoint calibration, $k$-corrections) are typically different. For that reason, and assuming that the spectroscopic data (either spectra or colors, but not both) are obtained under homogeneous conditions, it is safer to use the numbers displayed in Tables 2 and 3 in a differential way, i.e. as a guide to compare different diagrams within each of these tables, but not to compare the absolute values of the suitability indices between the two tables. The same reasoning makes the numbers displayed in Table 4 more uncertain that the ones shown in Tables 2 and 3.

Summarizing, and even considering all these problems, it is clear that once a given set of models has been adopted, the factor displayed in Eq. (22) is an excellent tool to estimate an optimized combination of spectroscopic measurements, in order to face the study of integrated spectra.

\section{Conclusions}

We have investigated the combined role of parameter degeneracy and signal-to-noise ratio in the study of the integrated spectroscopic properties of astronomical objects. In particular, we have examined the effect of random errors at the light of stellar population model predictions. We have shown that the expected 
Table 7. Comparison of $\log [\kappa]$ for the study of the age-metallicity degeneracy, computed from the prediction of three sets of models, W94 (Worthey 1994), V00 (Vazdekis et al. 1996; Blakeslee et al. 2001), and BC01 (Bruzual \& Charlot 2001). In all the cases we have derived the suitability parameters for SSP around 12 Gyr old and solar metallicity.

\begin{tabular}{|c|c|c|c|c|c|c|c|c|c|c|c|c|c|c|c|c|c|c|c|c|c|c|c|}
\hline index & models & \& & $\bar{Z}$ & $z^{N}$ & $\begin{array}{l}\hat{N} \\
\underset{J}{J}\end{array}$ & \begin{tabular}{l}
$\stackrel{8}{0}$ \\
\multirow{J}{*}{}
\end{tabular} & 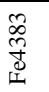 & $\begin{array}{l}\stackrel{n}{n} \\
\text { J } \\
\text { uj }\end{array}$ & $\begin{array}{l}\overrightarrow{\tilde{n}} \\
\text { fo } \\
\text { dit. }\end{array}$ & $\begin{array}{l}\infty \\
0 \\
0 \\
+ \\
0 \\
1\end{array}$ & 全 & $\begin{array}{l}n \\
0 \\
0 \\
0 \\
10\end{array}$ & $\bar{\Sigma}$ & $\sum^{\infty}$ & $\sum^{\infty}$ & 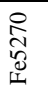 & $\begin{array}{l}n \\
m \\
\tilde{D} \\
1\end{array}$ & $\begin{array}{l}\text { o } \\
\text { ț } \\
0 \\
\text { L }\end{array}$ & 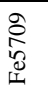 & 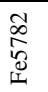 & $\begin{array}{l}\approx \\
\infty \\
\approx \\
\tilde{z}\end{array}$ & $\underset{Ð}{\check{0}}$ & $\overbrace{\mathscr{H}}^{\Omega}$ \\
\hline$\tilde{\mathrm{D}}_{4000}$ & W94 & $\ldots$ & 0.2 & 0.2 & 1.7 & 0.5 & 0.4 & 0.7 & 0.8 & 0.0 & 0.3 & 0.4 & 0.3 & 0.2 & 1.0 & 0.6 & 0.4 & 0.5 & 0.5 & 0.6 & 0.4 & 1.0 & 0.6 \\
\hline$\tilde{\mathrm{D}}_{4000}$ & V00 & $\ldots$ & 0.3 & 0.4 & 0.9 & 1.3 & 0.4 & 0.7 & 1.1 & 0.0 & 0.4 & 0.5 & 0.7 & 0.4 & 0.7 & 0.8 & 0.6 & 0.7 & 0.6 & 0.8 & 0.6 & 0.8 & 1.1 \\
\hline$\tilde{\mathrm{D}}_{4000}$ & BC01 & $\ldots$ & 0.2 & 0.4 & 1.2 & 1.9 & 0.3 & 0.6 & 0.8 & 0.0 & 0.5 & 0.4 & 0.2 & 0.0 & 0.5 & 0.6 & 0.4 & 0.5 & 0.6 & 0.7 & 0.3 & 1.4 & 0.9 \\
\hline $\mathrm{Fe} 4668$ & W94 & 0.0 & 1.1 & 1.2 & 1.2 & 1.1 & 1.3 & 1.6 & 1.7 & $\ldots$ & 1.2 & 2.2 & 1.0 & 0.8 & 1.2 & 1.7 & 1.9 & 1.9 & 2.4 & 3.0 & 1.3 & 1.6 & 1.4 \\
\hline $\mathrm{Fe} 4668$ & V00 & 0.0 & 0.9 & 0.9 & 1.1 & 1.3 & 1.2 & 1.7 & 1.5 & & 1.2 & 2.1 & & 0.7 & 1.3 & 1.5 & 1.7 & 1.7 & 3.1 & 2.2 & 1.1 & 1.6 & 1.3 \\
\hline $\mathrm{Fe} 4668$ & $\mathrm{BC} 01$ & 0.0 & 0.9 & 0.9 & 1.2 & 1.3 & 1.4 & 1.7 & 1.6 & $\cdots$ & 1.2 & 2.4 & 0.9 & 0.8 & 1.3 & 1.6 & 1.9 & 1.8 & 2.3 & 3.1 & 1.2 & 1.5 & 1.2 \\
\hline $\mathrm{Mg}_{2}$ & W94 & 0.2 & 1.5 & 1.4 & 1.4 & 1.0 & 1.5 & 1.9 & 2.2 & 0.8 & 0.9 & 1.3 & 2.0 & $\ldots$ & 1.6 & 1.6 & 1.3 & 1.4 & 1.3 & 1.4 & 1.5 & 1.5 & 2.0 \\
\hline $\mathrm{Mg}_{2}$ & V00 & 0.4 & 1.2 & 1.4 & 1.2 & 1.5 & 1.3 & 1.5 & 2.4 & 0.7 & 0.9 & 1.2 & 1.7 & $\ldots$ & 1.8 & 1.7 & 1.3 & 1.5 & 1.3 & 1.5 & 1.7 & 1.4 & 1.4 \\
\hline $\mathrm{Mg}_{2}$ & BC01 & 0.0 & 1.9 & 1.4 & 1.3 & 1.3 & 1.4 & 1.7 & 2.5 & 0.8 & 1.0 & 1.3 & 1.8 & $\ldots$ & 2.8 & 1.8 & 1.3 & 1.5 & 1.3 & 1.5 & 1.6 & 1.5 & 1.4 \\
\hline $\mathrm{Na} 5895$ & W94 & 0.4 & 2.1 & 2.6 & 1.6 & 1.3 & 3.1 & 2.9 & 2.6 & 1.3 & 1.3 & 1.8 & 1.7 & 1.5 & 1.7 & 2.4 & 2.0 & 2.1 & 1.8 & 1.9 & $\ldots$ & 1.8 & 2.2 \\
\hline Na5895 & V00 & 0.6 & 2.0 & 2.6 & 1.5 & 1.8 & 1.9 & 2.0 & 2.8 & 1.1 & 1.3 & 1.7 & 1.6 & 1.7 & 3.7 & 2.5 & 1.8 & 2.0 & 1.8 & 1.9 & $\ldots$ & 1.7 & 1.7 \\
\hline Na5895 & $\mathrm{BC} 01$ & 0.3 & 1.6 & 1.5 & 1.5 & 1.6 & 2.1 & 2.5 & 2.6 & 1.2 & 1.3 & 1.7 & 1.6 & 1.6 & 2.0 & 2.7 & 1.8 & 2.0 & 1.7 & 1.9 & $\ldots$ & 1.8 & 1.6 \\
\hline
\end{tabular}

random error in the measurement of line-strength indices and colors can be very easily computed as a constant divided by an appropriate average of the signal-to-noise ratio per $\AA$, as stated in Eq. (3). This simple expression allows to define a suitability parameter which combines both effects (degeneracy and sensitivity to noise), providing an immediate tool to compare the usefulness of different observational diagrams. The recipe to perform such a comparison is the following:

- (i) Chose a reliable stellar population model.

- (ii) Obtain the geometric transformation to convert the observational parameters (line-strength indices and colors) into physical data (age, metallicity, initial mass function,...). For this purpose, bivariate polynomial transformations (Appendix C) are a very convenient way to obtain the coefficients given in Eq. (C.2), or those corresponding to the inverse transformation, Eq. (19).

- (iii) Use Eq. (21) to obtain $\kappa$. The coefficients which indicate the sensitivity of the spectroscopic data to the signalto-noise ratio can be extracted from Table 1.

- (iv) Using reasonable estimates of the expected signal-tonoise ratio per $\AA$ in each spectroscopic measurement, employ Eq. (22) to obtain $\psi$. As discussed in Sect. 4.3, this is the factor to be minimized.

Classical atomic line-strength indices must be measured as molecular indices in order to apply the above procedure. The same also holds for generic indices (e.g. CaT, PaT, $\mathrm{CaT}^{*}$; see Cenarro et al. 2001).

We have illustrated this method by studying in more detail the well known age-metallicity degeneracy. Using model predictions for a 12 Gyr old simple stellar population with solar metallicity, we have shown that a broad spectral feature like the $\mathrm{D}_{4000}$ can be as well suited (or even more) than $\mathrm{H} \beta$ to analyze this kind of degeneracy, once the dependence on signalto-noise ratio is taken into account.

For all the reasons mentioned in Sect. 4.4, the aim of this paper is not to give a definite answer to the question of which is the best observational space to disentangle physical properties of stellar populations, but to provide an easy way to determine the relative suitability of different spectroscopic diagrams to obtain physical information of the astronomical objects under study.

Acknowledgements. Valuable discussions with A. Vazdekis, and J. J. González are gratefully acknowledged. The data employed in Appendix A was obtained with the $1 \mathrm{~m}$ JKT, $2.5 \mathrm{~m}$ INT and the 4.2m WHT at La Palma Observatory, and with the $3.5 \mathrm{~m}$ Telescope at Calar Alto Observatory. The JKT, INT and WHT are operated on the island of La Palma by the Royal Greenwich Observatory at the Observatorio del Roque de los Muchachos of the Instituto de Astrofísica de Canarias. The Calar Alto Observatory is operated jointly by the Max-Planck-Institute für Astronomie, Heidelberg, and the Spanish Comisión Nacional de Astronomía. This work was supported by the Spanish Programa Nacional de Astronomía y Astrofísica under grant AYA2000-977.

\section{References}

Aaronson, M., Cohen, J. G., Mould, J., \& Malkan, M. 1978, ApJ, 223, 824

Aragón-Salamanca, A., Gorgas, J., \& Rego, M., 1987, A\&A, 185, 97

Arimoto, N., \& Yoshii, Y. 1986, A\&A, 164, 260

Arimoto, N., \& Yoshii, Y. 1987, A\&A, 173, 23

Babu, G. J., \& Feigelson, E. D. 1996, Astrostatistics (London: Chapman \& Hall)

Bernardi, M., Sheth, R. K., Annis, J., et al. 2003, AJ, 125, 1817

Blakeslee, J. P., Vazdekis, A., \& Ajhar, E. A. 2001, MNRAS, 320, 193

Bressan, A., Chiosi, C., \& Fagotto, F. 1994, ApJS, 94, 63

Bruzual, G. 1983, ApJ, 273, 105

Bruzual, G. 2001, in IAU Symp. 207, Extragalactic Star Clusters, ed. E. K. Grebel, D.Geisler \& D. Minniti, IAU Symp. Ser., 207, in press [astro-ph/0110245]

Bruzual, G. 2002, in Proceedings of the XI Canary Islands Winter School of Astrophysics on Galaxies at High Redshifts, ed. I. Pérez-Fournon, \& F. Sánchez, Cambridge Contemporary Astrophysics, in press [astro-ph/0011094)]

Bruzual, G., \& Charlot, S. 1993, ApJ, 405, 538

Buzzoni, A. 1989, ApJS, 71, 817

Buzzoni, A. 1995, ApJS, 98, 69

Cardiel, N. 1999, Ph.D. thesis, Universidad Complutense de Madrid

Cardiel, N., Gorgas, J., Cenarro, J., \& González, J. J. 1998, A\&AS, 127,597 
Cardiel, N., Gorgas, J., Gallego, J., et al. 2002, SPIE, 4847, 297

Cenarro, A. J., Cardiel, N., Gorgas, J., et al. 2001, MNRAS, 326, 959

Cenarro, A. J., Gorgas, J., Cardiel, N., Vazdekis, A., \& Peletier, R. F. 2002, MNRAS, 329, 863

Cerviño, M., \& Mas-Hesse, J. M. 1994, A\&A, 284, 749

Cerviño, M., Luridiana, V., \& Castander, F. J. 2000, A\&A, 360, L5

Cerviño, M., Gómez, Flechoso, M. A., Castender, F. J., et al. 2001, A\&A, 376, 422

Cerviño, M., Valls-Gabaud, D., Luridiana, V., \& Mas-Hesse, J. M. 2002, A\&A, 381, 51

Cerviño, M., \& Valls-Gabaud, D. 2003, MNRAS, 338, 481

Charlot, S., \& Bruzual, G. 1991, ApJ, 367, 126

Charlot, S., Worthey, G., \& Bressan, A. 1996, ApJ, 457, 625

Chiosi, C., Bressan, A., \& Fagotto, F. 1996, A\&A, 311, 361

Crampin, J., \& Hoyle, F. 1961, MNRAS, 122, 27

Faber, S. M., Trager, S. C., González, J. J., \& Worthey, G. 1994, in Stellar Populations, ed. G. Gilmore \& P. van der Kruit (Dordrecht: Kluwer), IAU Symp., 164, 249

Fioc, M., \& Rocca-Volmerange, B. 1997, A\&A, 326, 950

Fritze-v. Alvensleben, U. A., \& Gerhard, O. E. 1994, A\&A, 285, 571

Fukugita, M., Shimasaku, K., \& Ichikawa, T. 1995, PASP, 107, 945

Gorgas, J., Faber, S. M., Burstein, D., et al. 1993, ApJS, 86, 153

Gorgas, J., Cardiel, N., Pedraz, S., \& González, J. J. 1999, A\&AS, 139,29

Guiderdoni, B., \& Rocca-Volmerange, B. 1987, A\&A, 186, 1

Gunn, J. E., Stryker, L. L., \& Tinsley, B. 1981, ApJ, 249, 48

Hammer, F., Flores, H., Lilly, S. J., et al. 1997, ApJ, 481, 49

Jones, L. A., \& Worthey, G. 1995, ApJ, 446, L31

Kauffmann, G., Heckman, T. M., White, S. D. M., et al. 2003, MNRAS, 341, 33

Kendall, M. G. 1961, A Course in the Geometry of $n$ Dimensions, Griffin's Statistical Monographs and Courses \#8, London

Koo, D. C. 1995, in Wide Field Spectroscopy and the Distant Universe, eds. S. Maddox and A. Aragón-Salamanca (Singapore: World Scientific), 55

Kuntschner, H., Lucey, J. R., Smith, J., Hudson, M. J., \& Davies, R. L. 2001, MNRAS, 323, 615

Le Fèvre, O., Saisse, M., Mancini, D., et al. 2000, SPIE, 4008, 546

Leitherer, C., \& Heckman, T. M. 1995, ApJS, 96, 9

Leitherer, C., Alloin, D., Fritze, V., Alvensleben, H., et al. 1996, PASP, 108, 996

Leitherer, C., Schraerer, D., Goldader, J. D., et al. 1999, ApJS, 123, 3

Lilly, S. J., Le Fèvre, O., Crampton, D., Hammer, F., \& Tresse, L. 1995, ApJ, 455, 50
Maraston, C. 1998, MNRAS, 300, 872

García-Vargas, M. L., Mollá, M., \& Bressan, A. 1998, A\&AS, 130, 513

Mas-Hesse, J. M., \& Kunth, D. 1991, A\&AS, 88, 399

Mayya, Y. D. 1995, AJ, 109, 2503

Mayya, Y. D. 1997, ApJ, 482, L149

Milone, A., Barbuy, B., \& Bica, E. 1995, A\&AS, 113, 547

Mollá, M., \& García-Vargas, M. L. 2000, A\&A, 359, 18

Mould, J. 1993, in Sky Surveys: Protostars to Protogalaxies, ed. B. T. Soifer, ASP Conf. Ser., 43, 281

O'Connell, R. W. 1976, ApJ, 206, 370

O'Connell, R. W. 1980, ApJ, 236, 430

O'Connell, R. W. 1994, in Nuclei of Normal Galaxies: Lessons from the Galactic Center, ed. R. Genzel (Dordrecht: Kluwer), 255

Origlia, L., \& Oliva, E. 2000, A\&A, 357, 610

Pickles A. J. 1998, PASP, 110, 863

Rose, J. A. 1985, AJ, 90, 1927

Rose, J. A. 1994, AJ, 107, 206

Schiavon, R. P., Barbuy, B., \& Bruzual, G. 2000, ApJ, 532, 453

Tantalo, R., Chiosi, C., Bressan, A., Marigo, P., \& Portinari, L. 1998, A\&A, 335, 823

Thomas, D., Maraston, C., \& Bender, R. 2003, MNRAS, 339, 897

Tinsley, B. 1972, ApJ, 178, 319

Tinsley, B. 1978, ApJ, 222, 14

Tinsley, B. 1980, Fund. Cos. Phys., 5, 287

Tinsley, B., \& Gunn, J. E. 1976, ApJ, 203, 52

Vazdekis, A. 1999, ApJ, 513, 224

Vazdekis, A., \& Arimoto, N. 1999, 525, 144

Vazdekis, A., Casuso, E., Peletier, R. F., \& Beckman, J. E. 1996, ApJS, 106, 307

Vazdekis, A., Peletier, R. F., Beckman, J. E., \& Casuso, E. 1997, ApJS, 111, 203

Vazdekis, A., Cenarro, A. J., Gorgas, J., Cardiel, N., \& Peletier, F. F. 2003, MNRAS, 340, 1317

Wegner, G., Colless, M., Baggley, G., et al. 1996, ApJS, 106, 1

Worthey, G. 1994, ApJS, 95, 107

Worthey, G., Faber, S. M., González, J. J., \& Burstein, D. 1994, ApJS, 94, 687

Worthey, G., \& Ottaviani. D. L. 1997, ApJS, 111, 377

York, D. G., Adelman, J., Anderson, J. E., et al. 2000, AJ, 120, 1579

Zackrisson, E., Bergvall, N., Olofsson, K., \& Siebert, A. 2001, A\&A, 375,814 
N. Cardiel et al.: Using spectroscopic data to disentangle stellar population properties, Online Material p 1

\section{Online Material}


N. Cardiel et al.: Using spectroscopic data to disentangle stellar population properties, Online Material p 2

\section{Appendix A: Estimating random errors in the $D_{4000}$}

In order to explore in more detail the validity of Eq. (4), we have compared the error estimations in the $\mathrm{D}_{4000}$ derived from that formulae with the results derived by using a more accurate method.

In Fig. A.1 we represent the relative errors in the $\mathrm{D}_{4000}$, $\epsilon_{\mathrm{r}}\left[\mathrm{D}_{4000}\right]$, as a function of the signal-to-noise per $\AA$, as measured in the 713 spectra (including repeated observations) of the stellar library gathered by Gorgas et al. (1999) to derive the empirical calibration of this spectral feature. In panel a we present the measured relative error determined using an accurate method - Eqs. (38)-(40) of Cardiel et al. (1998) -, for four different estimations of the signal-to-noise ratio per $\AA$. In particular, crosses and small dots indicate the results obtained when using the mean $S / N(\AA)$ in the blue and red bandpasses, respectively; the signal-to-noise ratio for the open circles has been computed as the arithmetic mean of the two previous values, whereas for the filled circles we have employed the weighted mean $\left(1 /\langle S / N\rangle_{\text {blue }}+\right.$ $\left.1 /\langle S / N\rangle_{\text {red }}\right) /\left(1 /\langle S / N\rangle_{\text {blue }}^{2}+1 /\langle S / N\rangle_{\text {red }}^{2}\right)$. The prediction of Eq. (4) is the diagonal full line, whereas the residuals with respect to this prediction are plotted in panel $b$. The employed stellar sample typically contains spectra with poorer signal-tonoise ratio in the blue bandpass of the $D_{4000}$ than in the red bandpass, and the simple arithmetic mean of the $S / N(\AA)$ is not a good approximation.

It is clear from the previous figure that the weighted mean is the best approximation. We have also checked that a weighted mean of the form $\left[2 /\left(1 /\langle S / N\rangle_{\text {blue }}^{2}+1 /\langle S / N\rangle_{\text {red }}^{2}\right)\right]^{1 / 2}$ (not shown in the figure) gives acceptable results.

\section{Appendix B: Estimating random errors in colors}

With the aim of estimating the dependence of random errors in colors on the signal-to-noise ratio, we are following here a similar procedure to that employed in Cardiel et al. (1998) to derive the corresponding formulae for classical indices.

Given two filters, a spectral energy distribution $f(\lambda)$, and the SED of a reference object $\mathcal{F}(\lambda)$ (e.g. the SED of $\alpha \mathrm{Lyr}$ for magnitudes measured in the Vega system, $\mathcal{F}(v)=3.63 \times$ $10^{-20} \mathrm{erg} \mathrm{cm}^{-2} \mathrm{~s}^{-1} \mathrm{~Hz}^{-1}$ for $\mathrm{AB}$ magnitudes, or $\mathcal{F}(\lambda)=3.63 \times$ $10^{9} \mathrm{erg} \mathrm{cm}^{-2} \mathrm{~s}^{-1} \AA^{-1}$ for HST magnitudes), a color can be determined by (see e.g. Fukugita et al. 1995)

$$
\begin{aligned}
C= & -2.5 \log _{10}\left[\frac{\int_{\lambda} f(\lambda) R_{1}(\lambda) \mathrm{d} \lambda}{\int_{\lambda} f(\lambda) R_{2}(\lambda) \mathrm{d} \lambda}\right] \\
& +2.5 \log _{10}\left[\frac{\int_{\lambda} \mathcal{F}(\lambda) R_{1}(\lambda) \mathrm{d} \lambda}{\int_{\lambda} \mathcal{F}(\lambda) R_{2}(\lambda) \mathrm{d} \lambda}\right],
\end{aligned}
$$

where $R_{i}(\lambda)$ is the response function of the $i$ th filter. The previous expression can be rewritten as

$$
C=-2.5 \log _{10} \frac{\langle f(\lambda)\rangle_{1}}{\langle f(\lambda)\rangle_{2}}+C_{0},
$$

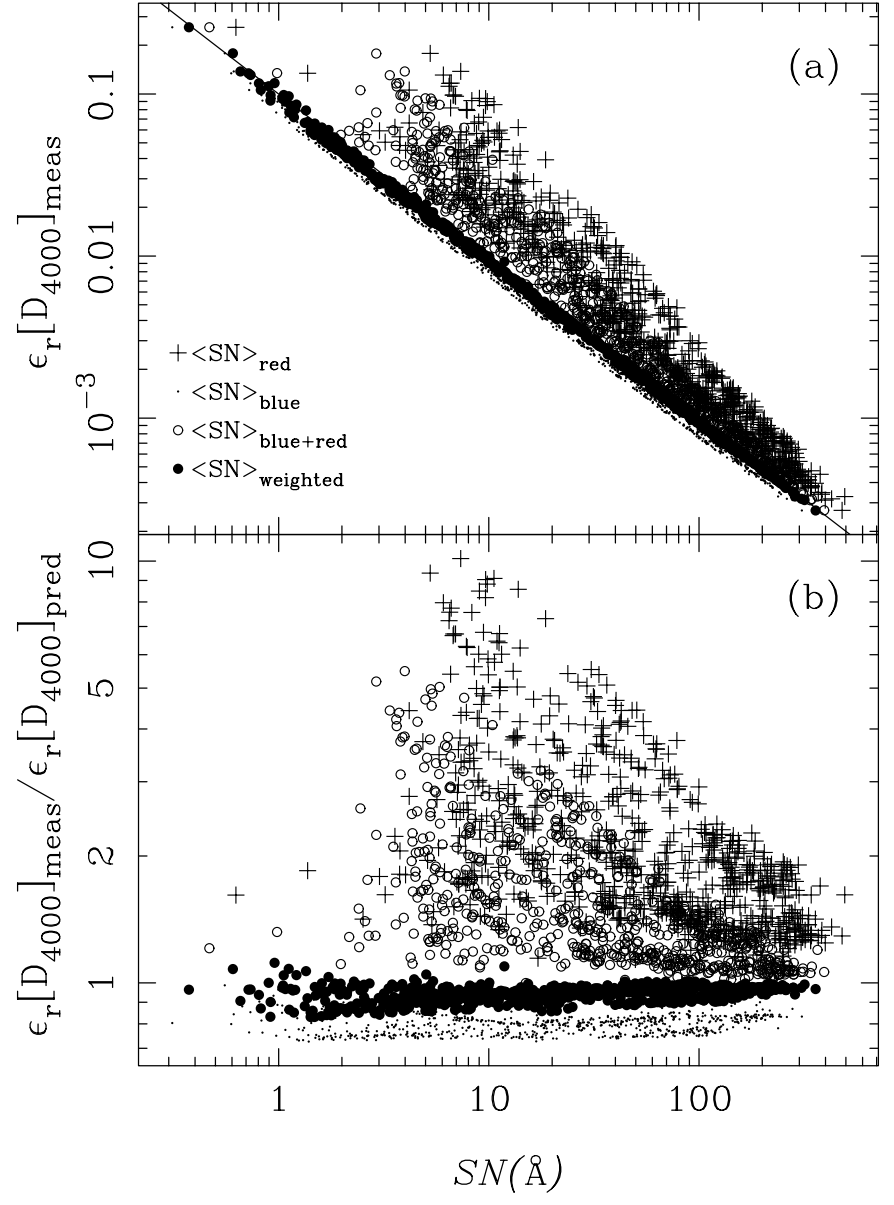

Fig. A.1. Relative errors in the $\mathrm{D}_{4000}, \epsilon_{\mathrm{r}}\left[\mathrm{D}_{4000}\right]$, as a function of the signal-to-noise per $\AA$, measured in the stellar library employed by Gorgas et al. (1999) to derive the empirical calibration of this spectral feature. See text in Appendix A for details.

where

$\langle f(\lambda)\rangle_{i}=\frac{\int_{\lambda} f(\lambda) R_{i}(\lambda) \mathrm{d} \lambda}{\int_{\lambda} R_{i}(\lambda) \mathrm{d} \lambda}$,

$C_{0}=-2.5 \log _{10} \frac{k_{1}}{k_{2}}+\tilde{C_{0}}$

$k_{i}=\int_{\lambda} R_{i}(\lambda) \mathrm{d} \lambda$

and

$\tilde{C}_{0}=+2.5 \log _{10}\left[\frac{\int_{\lambda} \mathcal{F}(\lambda) R_{1}(\lambda) \mathrm{d} \lambda}{\int_{\lambda} \mathcal{F}(\lambda) R_{2}(\lambda) \mathrm{d} \lambda}\right]$.

In practice integrals must be replaced by summations of the form

$\int_{\lambda} f(\lambda) R_{i}(\lambda) \mathrm{d} \lambda \simeq \Theta \sum_{k=1}^{N_{i}} f\left(\lambda_{i, k}\right) R_{i}\left(\lambda_{i, k}\right)$, 
N. Cardiel et al.: Using spectroscopic data to disentangle stellar population properties, Online Material p 3

where $\Theta$ is the linear dispersion (in $\AA$ pixel ${ }^{-1}$, assumed to be constant along the spectrum), $N_{i}$ is the number of pixels covering the $i$ th filter, $\lambda_{i, k}$ is the central wavelength of the $k$ th pixel of the $i$ th filter, and $f\left(\lambda_{i, k}\right)$ is the flux is that pixel.

If $\sigma_{f}(\lambda)$ is the error spectrum associated with $f(\lambda)$, and if we assume that $\mathcal{F}(\lambda)$ and $R_{i}(\lambda)$ are error free, the expected error in the color can be expressed as

$\sigma(C)=\frac{2.5 \log _{10} e}{\frac{\langle f(\lambda)\rangle_{1}}{\langle f(\lambda)\rangle_{2}}} \sigma\left(\frac{\langle f(\lambda)\rangle_{1}}{\langle f(\lambda)\rangle_{2}}\right)$.

The square of the last term in the previous expression can be written as

$\sigma^{2}\left(\frac{\langle f(\lambda)\rangle_{1}}{\langle f(\lambda)\rangle_{2}}\right)$

$$
=\frac{\sigma^{2}\left(\langle f(\lambda)\rangle_{1}\right)}{\left(\langle f(\lambda)\rangle_{2}\right)^{2}}+\frac{\left(\langle f(\lambda)\rangle_{1}\right)^{2}}{\left(\langle f(\lambda)\rangle_{2}\right)^{2}} \frac{\sigma^{2}\left(\langle f(\lambda)\rangle_{2}\right)}{\left(\langle f(\lambda)\rangle_{2}\right)^{2}} .
$$

In the other hand, from Eq. (B.2) it is immediate to show that

$$
\frac{\left(\langle f(\lambda)\rangle_{1}\right)^{2}}{\left(\langle f(\lambda)\rangle_{2}\right)^{2}}=\left[10^{-0.4\left(C-C_{0}\right)}\right]^{2}
$$

The first fraction in the right hand side of Eq. (B.9) can also be rewritten as

$$
\begin{aligned}
\frac{\sigma^{2}\left(\langle f(\lambda)\rangle_{1}\right)}{\left(\langle f(\lambda)\rangle_{2}\right)^{2}} & =\frac{\left(\langle f(\lambda)\rangle_{1}\right)^{2}}{\left(\langle f(\lambda)\rangle_{2}\right)^{2}} \frac{\sigma^{2}\left(\langle f(\lambda)\rangle_{1}\right)}{\left(\langle f(\lambda)\rangle_{1}\right)^{2}} \\
& =\left[10^{-0.4\left(C-C_{0}\right)}\right]^{2} \frac{\sigma^{2}\left(\langle f(\lambda)\rangle_{1}\right)}{\left(\langle f(\lambda)\rangle_{1}\right)^{2}} .
\end{aligned}
$$

It is not difficult to show that assuming that the error spectrum is roughly constant within each bandpass filter, making use of Eqs. (B.3) and (B.5), and after replacing the integrals by summations,

$\frac{\sigma^{2}\left(\langle f(\lambda)\rangle_{i}\right)}{\left(\langle f(\lambda)\rangle_{i}\right)^{2}} \simeq\left(\frac{\left\langle\sigma_{i, \AA}\right\rangle}{\langle f(\lambda)\rangle_{i, \AA}}\right)^{2} \xi_{i} \simeq \frac{1}{\left[S / N(\AA)_{i}\right]^{2}} \xi_{i}$,

where $\langle f(\lambda)\rangle_{i, \AA}$ and $\left\langle\sigma_{i, \AA}\right\rangle$ are the mean flux and mean error per $\AA$ in the $i$ th filter, respectively, $S / N(\AA)_{i}$ the mean signal-tonoise ratio per $\AA$ in the $i$ th filter, and

$\xi_{i} \equiv \frac{1}{\Theta} \frac{\sum_{k=1}^{N_{i}}\left[R_{i}\left(\lambda_{i, k}\right)\right]^{2}}{\left[\sum_{k=1}^{N_{i}} R_{i}\left(\lambda_{i, k}\right)\right]^{2}}$.

Introducing the result of Eqs. (B.9)-(B.12) into Eq. (B.8), we obtain

$$
\begin{aligned}
\sigma(C)= & 2.5 \log _{10} e \\
& \times\left(\frac{\xi_{1}}{\left[S / N(\AA)_{1}\right]^{2}}+\frac{\xi_{2}}{\left[S / N(\AA)_{2}\right]^{2}}\right)^{1 / 2} .
\end{aligned}
$$

If $S / N(\AA)_{1} \approx S / N(\AA)_{2}$, this last expression adopts the same form that Eq. (3),

$\sigma(C) \simeq \frac{c(C)}{S / N(\AA)}$

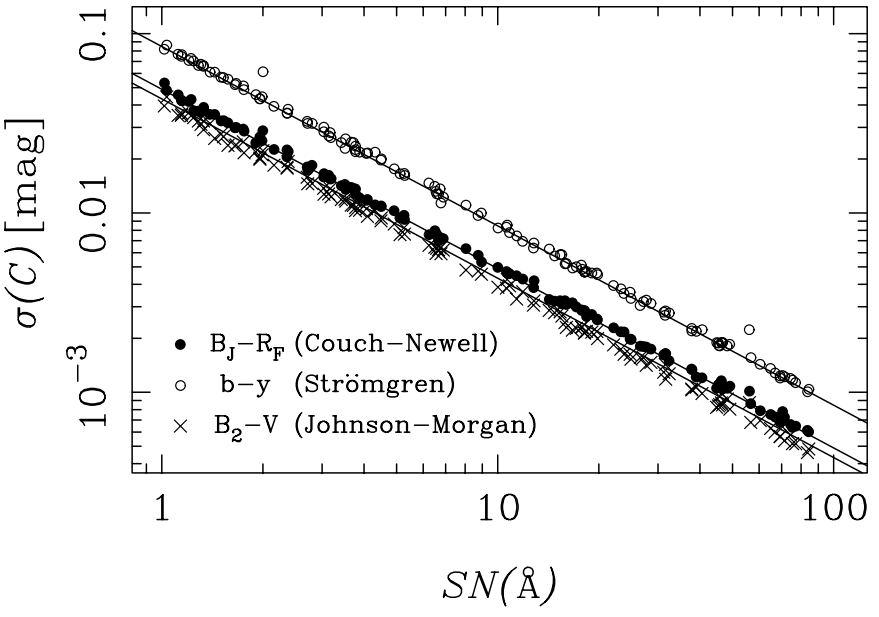

Fig. B.1. Random errors from numerical simulations in the measurement of three colors in the 131 stellar spectra from the library of Pickles (1998), as a function of the signal-to-noise ratio per $\AA$. The full lines are the predictions of Eq. (B.16). See text for details.

where

$$
\begin{aligned}
c(C) & =2.5 \log _{10} e \times\left(\xi_{1}+\xi_{2}\right)^{1 / 2} \\
& \simeq 1.086\left(\xi_{1}+\xi_{2}\right)^{1 / 2} .
\end{aligned}
$$

Numerical values of $\xi_{i}$ for typical photometric bands are given in Table B.1.

For illustration, we compare in Fig. B.1 the predictions of Eq. (B.16), for three sample colors, with the results of numerical simulations. For this purpose, we have employed the 131 stellar spectra from the library of Pickles (1998), which contains SEDs with an ample range of spectral types and luminosity classes. For each of these spectra, we have built a synthetic error spectrum by randomly choosing a given $S / N(\AA)$. Color errors were then measured in simulated instances of the spectra generated with Eq. (2). The full lines in Fig. B.1 are not fits to the data points, but the predictions of Eq. (B.16) using the corresponding $\xi_{i}$ parameters (extracted from Table B.1).

Finally, it is also possible to express the $\xi_{i}$ coefficients as a function of the filter width. In fact Eq. (B.13) is the discrete expression of the more general definition

$\xi_{i} \equiv \frac{\int_{\text {filter }_{i}} R(\lambda)^{2} \mathrm{~d} \lambda}{\left[\int_{\text {filter }_{i}} R(\lambda) \mathrm{d} \lambda\right]^{2}}$.

If now we assume that the filter transmission can be approximately described by a box function of the form

$R(\lambda)= \begin{cases}R_{0} \text { (constant) } & \text { for } \lambda_{1} \leq \lambda \leq \lambda_{2}, \\ 0 & \text { for } \lambda<\lambda_{1} \text { or } \lambda>\lambda_{2},\end{cases}$

it is immediate to show that

$\xi=\left(\lambda_{2}-\lambda_{1}\right)^{-1} \equiv F W(\AA)^{-1}$,

where $F W(\AA)$ is the filter width in $\AA$. In practice, since filter response functions are not exactly box functions, filter widths can be approximately computed as

$F W(\AA) \simeq \int_{\text {filter }_{i}} \tilde{R}(\lambda) \mathrm{d} \lambda$, 
N. Cardiel et al.: Using spectroscopic data to disentangle stellar population properties, Online Material p 4

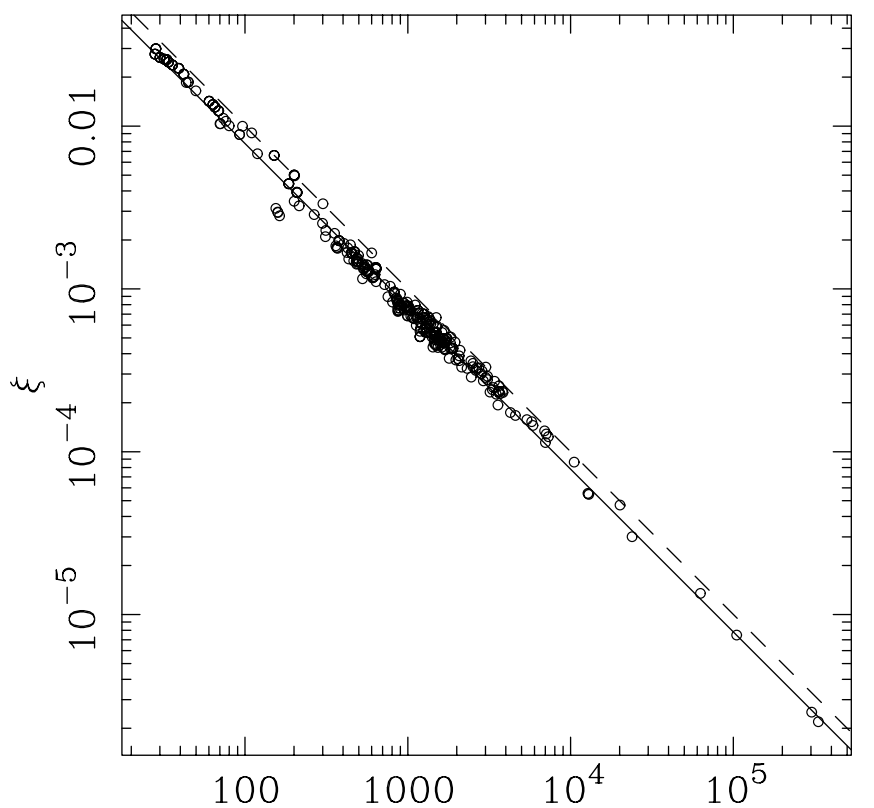

Filter width $(\AA)$

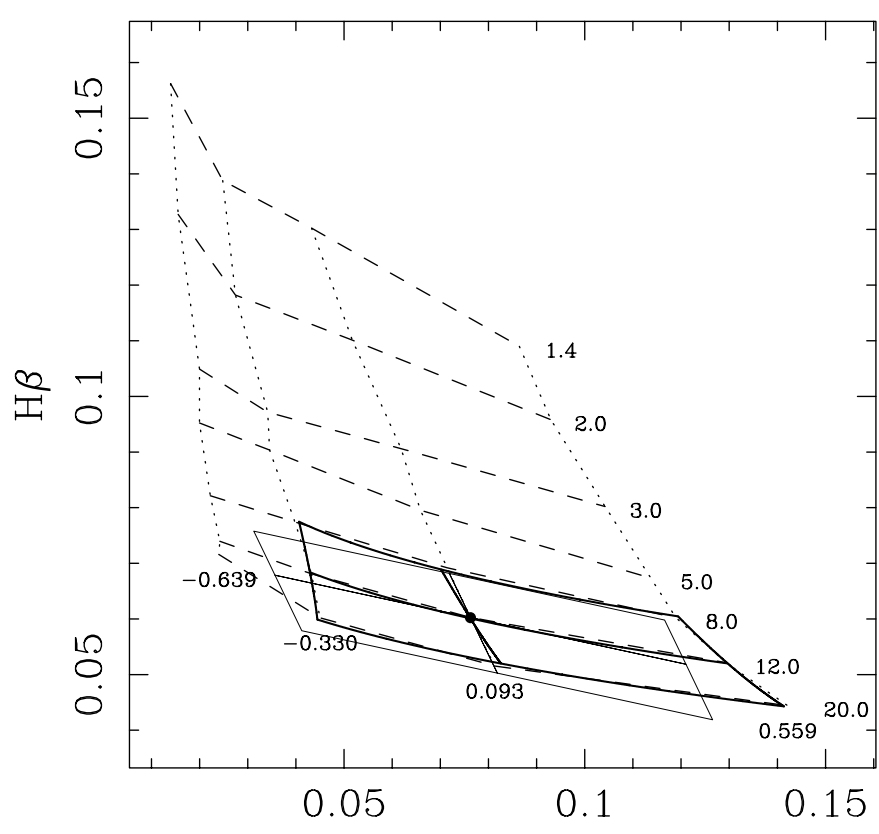

Fe4668

Fig. B.2. Comparison of the $\xi$ values as a function of the filter width, computed with Eqs. (B.13) and (B.20), respectively. The measurements have been performed using the response function of a set of 360 photometric bands collected from the literature (including those which are incorporated in the source code of the GISSEL models, and HST, KPNO and ESO filters, among others). The solid line is Eq. (B.21), a least square fit to the data, and the dashed line is the prediction of Eq. (B.19). The difference between both lines is due to the fact that filter response curves are not exactly box functions.

being $\tilde{R}(\lambda)$ the response function normalized to unity. To check the validity of these approximations, in Fig. B.2 we graphically compare the measured filter widths versus the $\xi$ values derived using Eq. (B.13), for a collection of 360 filter response functions collected from the literature (see table caption). The full line is a least squares fit, whereas the dashed line is the prediction of Eq. (B.19). As it is apparent from the figure, there is an excellent correlation between the $\xi$ coefficients and the filter width, although the linear fit to the data points (full line) indicates that, on average,

$\xi \simeq(0.78 \pm 0.09) F W(\AA)^{-1}$,

where the error in the coefficient is the residual standard deviation around the fit.

\section{Appendix C: Measuring age and metallicity from index-index diagrams}

Iso-metallicity and iso-age lines in index-index, index-color and color-color diagrams are usually far from displaying very regular grids, but they typically exhibit unevenly spaced and distorted patterns. For this reason, the computation of ages and metallicities from a given pair of spectroscopic measurements should be addressed through the use of local mapping functions which properly accounts for the geometric distortions.

Fig. C.1. Example of fit of local polynomials to describe the local behavior of line-strength indices as a function of physical parameters. Dashed and dotted lines are the predictions of Bruzual \& Charlot (2001) models for single stellar populations of fixed age and metallicity, respectively (ages are given in Gyr, and metallicities as $[\mathrm{Fe} / \mathrm{H}]$ ). The thin solid lines indicates the resulting fit after using Eq. (C.1) with $N=1$, around the model predictions for a SSP of 12 Gyr and solar metallicity (the coefficients were determined using a least-squares fit to 5 points: the grid model point chosen as the origin of the local transformation, and the two closest points in both age and metallicity). It is clear that the fit can not be extrapolated very far from the central point. The thick solid lines show the result for $N=2$ (derived from the fit to the 9 points: the 5 points previously employed in the fit for $N=1$, plus the 4 additional corners of the region delineated by the thick line). In this case it is clear that the $N=2$ polynomial approximation provides a very reasonable representation of the geometric distortions when moving from the observational to the physical parameter space.

Obviously, the same is also true for the computation of the local derivatives (i.e. metal sensitivity parameters).

An excellent approach to this problem is the use of bivariate polynomial transformations of the form (see e.g. Wolberg 1992)

$m_{1}=\sum_{i=0}^{N} \sum_{j=0}^{N-i} p_{i j}(\log [\operatorname{age}])^{i}(\log [Z])^{j}$,
$m_{2}=\sum_{i=0}^{N} \sum_{j=0}^{N-i} q_{i j}(\log [\operatorname{age}])^{i}(\log [Z])^{j}$,

where $N$ is the polynomial degree, and $p_{i j}$ and $q_{i j}$ are the polynomial coefficients. The inverse transformation can be written by an analogous expression.

For $N=1$ the above equations only account for affine transformations (i.e. translation, rotation, scale and shear). 
N. Cardiel et al.: Using spectroscopic data to disentangle stellar population properties, Online Material p 5

Table B.1. Numerical values of the $\xi$ coefficients computed from Eq. (B.13), for a set of common photometric bands corresponding to the filters given in Table 9 of Fukugita et al. (1995). Filter widths were determined with Eq. (B.20).

\begin{tabular}{|c|c|c|c|c|c|c|c|}
\hline$\overline{\text { Bandpass system }}$ & Band & $\begin{array}{l}\text { Width } \\
(\AA)\end{array}$ & $\begin{array}{c}\xi \\
\times 10^{-4}\end{array}$ & Bandpass system & Band & $\begin{array}{l}\text { Width } \\
(\AA)\end{array}$ & $\begin{array}{c}\xi \xi \\
\times 10^{-4}\end{array}$ \\
\hline \multirow[t]{4}{*}{ Johnson-Morgan } & $\bar{U}$ & 543 & 13.65 & \multirow{4}{*}{$\begin{array}{l}\text { Schneider et al. } \\
\text { (4-shooter) }\end{array}$} & $g_{4}$ & 943 & 8.17 \\
\hline & $B_{2}$ & 1010 & 7.70 & & $r_{4}$ & 906 & 7.97 \\
\hline & $V$ & 871 & 8.15 & & $i_{4}$ & 1405 & 5.06 \\
\hline & & & & & $z_{4}$ & 1286 & 5.41 \\
\hline \multirow[t]{3}{*}{ Cousins } & $R_{\mathrm{C}}$ & 1591 & 4.56 & \multirow{5}{*}{$\begin{array}{l}\text { Schneider et al. } \\
\text { (Pfuei) }\end{array}$} & & & \\
\hline & $I_{\mathrm{C}}$ & 1495 & 5.82 & & $g$ & 894 & 7.60 \\
\hline & & & & & $r$ & 880 & 7.90 \\
\hline \multirow[t]{2}{*}{ Johnson } & $R_{\mathrm{J}}$ & 1978 & 3.66 & & $i$ & 1196 & 5.73 \\
\hline & $I_{\mathrm{J}}$ & 2148 & 3.32 & & $z$ & 1187 & 5.50 \\
\hline \multirow[t]{4}{*}{ Sandage-Smith } & $u$ & 580 & 12.85 & \multirow{4}{*}{$\begin{array}{l}\text { Schneider et al. } \\
\text { (narrow bands) }\end{array}$} & $A$ & 498 & 16.01 \\
\hline & $b$ & 1046 & 7.34 & & $B$ & 443 & 18.68 \\
\hline & $v$ & 854 & 8.76 & & $C$ & 571 & 13.17 \\
\hline & $r$ & 998 & 6.84 & & $D$ & 491 & 15.40 \\
\hline \multirow[t]{5}{*}{ Strömgren } & $u$ & 355 & 21.95 & \multirow[t]{4}{*}{ Tyson (CCD) } & $B_{\mathrm{J}}$ & 1214 & 6.16 \\
\hline & $v$ & 200 & 34.49 & & $R$ & 1307 & 6.42 \\
\hline & $b$ & 215 & 32.36 & & $I$ & 1688 & 4.30 \\
\hline & $y$ & 266 & 28.57 & & & & \\
\hline & & & & \multirow[t]{5}{*}{ WFPC2 } & F555W & 1489 & 5.13 \\
\hline \multirow[t]{5}{*}{ Kron } & $U_{\mathrm{K}}$ & 565 & 12.72 & & F606W & 1849 & 4.32 \\
\hline & $J_{\mathrm{K}}$ & 1297 & 5.74 & & F702W & 1662 & 4.72 \\
\hline & $F_{\mathrm{K}}$ & 1190 & 5.09 & & F814W & 1485 & 4.52 \\
\hline & $N_{\mathrm{K}}$ & 1646 & 4.98 & & & & \\
\hline & & & & \multirow[t]{3}{*}{ POSS II } & $g_{\text {POSS }}$ & 780 & 10.40 \\
\hline \multirow[t]{2}{*}{ Couch-Newell } & $B_{\mathrm{J}}$ & 1384 & 6.10 & & $r_{\mathrm{POSS}}$ & 991 & 7.47 \\
\hline & $R_{\mathrm{F}}$ & 486 & 14.23 & & $i_{\mathrm{POSS}}$ & 1191 & 6.25 \\
\hline \multirow[t]{5}{*}{ Thuan-Gunn } & $u$ & 404 & 19.02 & \multirow[t]{5}{*}{ SDSS } & $u^{\prime}$ & 568 & 13.92 \\
\hline & $v$ & 485 & 15.33 & & $g^{\prime}$ & 1264 & 6.66 \\
\hline & $g$ & 722 & 10.63 & & $r^{\prime}$ & 1333 & 6.76 \\
\hline & $r$ & 901 & 9.27 & & $i^{\prime}$ & 1349 & 6.08 \\
\hline & & & & & $z^{\prime}$ & 1309 & 5.39 \\
\hline
\end{tabular}

This linear approximation is valid when the 6 polynomial coefficients are derived from control points (those for which line-strength indices, ages and metallicities are given by the models) which are very close to the point $\left(p_{00}, q_{00}\right)$. Although most evolutionary synthesis models provide these close control points when considering the line-strength predictions as a function of age, the same is not true for the indices variations as a function of metallicity. This problem leads to systematic uncertainties in the index predictions, as shown in Fig. C.1 for the $\mathrm{H} \beta$-Fe4668 diagram.

The second-degree approximation, $N=2$, improves the quality of the prediction allowing for a selection of more distant control points. In this case, 12 coefficients must be computed by solving two systems of 6 linear equations. It is straightforward to show that the coefficients of the A matrix in Eq. (16) can be rewritten as a function of the bivariate polynomial coefficients as

$a_{11}=p_{10}+p_{11} \log [$ age $]+2 p_{20} \log [Z]$,

$a_{12}=p_{01}+p_{11} \log [Z]+2 p_{02} \log [$ age $]$,

$a_{21}=q_{10}+q_{11} \log [$ age $]+2 q_{20} \log [Z]$

$a_{22}=q_{01}+q_{11} \log [Z]+2 q_{02} \log [$ age $]$.

In Fig. C. 1 we also show the mapping obtained for $N=2$ (thick solid lines), which clearly improves the result obtained for $N=1$. Higher order polynomials $(N=3, \ldots)$ are typically unnecessary, since the required number of points to perform the fit increases rapidly, and in these situations it is always possible to constraint the fit to a smaller region with $N=2$.

Obviously, the same procedure is valid when reading other physical parameters from other grids predicted by stellar population models. 